\title{
The origin and evolution of a two- component system of paralogous genes encoding the centromeric histone CENH3 in cereals
}

\author{
Evgeny A. Elisafenko ${ }^{1,2}$, Elena V. Evtushenko ${ }^{2}$ and Alexander V. Vershinin ${ }^{2,3^{*}}$
}

\begin{abstract}
Background: The cereal family Poaceae is one of the largest and most diverse angiosperm families. The central component of centromere specification and function is the centromere-specific histone H3 (CENH3). Some cereal species (maize, rice) have one copy of the gene encoding this protein, while some (wheat, barley, rye) have two. We applied a homology-based approach to sequenced cereal genomes, in order to finally trace the mutual evolution of the structure of the CENH3 genes and the nearby regions in various tribes.
\end{abstract}

Results: We have established that the syntenic group or the CENH3 locus with the CENH3 gene and the boundaries defined by the CDPK2 and bZIP genes first appeared around 50 Mya in a common ancestor of the subfamilies Bambusoideae, Oryzoideae and Pooideae. This locus came to Pooideae with one copy of CENH3 in the most ancient tribes Nardeae and Meliceae. The $\beta C E N H 3$ gene as a part of the locus appeared in the tribes Stipeae and Brachypodieae around 35-40 Mya. The duplication was accompanied by changes in the exon-intron structure. Purifying selection acts mostly on aCENH3s, while $\beta C E N H 3$ s form more heterogeneous structures, in which clade-specific amino acid motifs are present. In barley species, the $\beta C E N H 3$ gene assumed an inverted orientation relative to aCENH3 and the CDPK2 gene was substituted with $\angle H C B-I$. As the evolution and domestication of plant species went on, the locus was growing in size due to an increasing distance between aCENH3 and $\beta C E N H 3$ because of a massive insertion of the main LTR-containing retrotransposon superfamilies, gypsy and copia, without any evolutionary preference on either of them. A comparison of the molecular structure of the locus in the A, B and D subgenomes of the hexaploid wheat $T$. aestivum showed that invasion by mobile elements and concomitant rearrangements took place in an independent way even in evolutionarily close species.

Conclusions: The CENH3 duplication in cereals was accompanied by changes in the exon-intron structure of the $\beta C E N H 3$ paralog. The observed general tendency towards the expansion of the CENH3 locus reveals an amazing diversity of ways in which different species implement the scenario described in this paper.

Keywords: Centromere, CENH3, Gene duplication, Molecular evolution, Invasion of transposable elements, Poaceae

*Correspondence: avershin@mcb.nsc.ru

${ }^{3}$ Novosibirsk State University, Novosibirsk 630090, Russia

Full list of author information is available at the end of the article

\section{Background}

Histone $\mathrm{H} 3$ is one of the four histone proteins that compose nucleosome cores. Canonical histone $\mathrm{H} 3$ has a globular C-terminal domain (HFD, the histone-fold domain), which harbors four helix motifs $(\alpha \mathrm{N}, \alpha 1, \alpha 2, \alpha 3)$ and the loop1 and loop2 regions. A putative recognition site for 
histone chaperones involved in nucleosome assembly partially overlaps with the $\alpha 2$ helix [1]. The $\mathrm{N}$-terminal domain (NTT, N-terminal tail) is considered to function in the formation of higher-order chromatin, and deletion of the H3 NTT affects histone-DNA interactions and substantially decreases nucleosome stability [2]. The specific role of histone $\mathrm{H} 3$ is that inclusion of one of its variants (the one designated as CENP-A in animals and as CENH3 (centromere-specific histone $\mathrm{H} 3$ ) in plants) in the nucleosomes defines the location of centromeres on chromosomes [3] and is essential for centromere and kinetochore formation in most organisms [4]. Within the HFD, loop 1 and $\alpha 2$ helix are necessary for targeting centromeric histone $\mathrm{H} 3$ to the centromere and the region formed by the two is referred to as the CATD [5]. The crystal structure of the nucleosome revealed that CENPA structurally differs from conventional histone $\mathrm{H} 3$ at the $\mathrm{N}$-terminal region in the nucleosome and this specific structure may cause the unwrapping of DNA at the entry/ exit regions in the nucleosome [6]. Overall, the DNA wraps less tightly in centromeric than in conventional nucleosomes and these differences suggest that they are distinct from canonical $\mathrm{H} 3$ nucleosomes $[7,8]$. The histone $\mathrm{H} 3$ family comprises at least three canonic $\mathrm{H} 3$ variants in most eukaryotes and these members, H3.1, H3.2, and $\mathrm{H} 3.3$, share more than $95 \%$ identity, while CENH3s are highly divergent in sequence compared to canonical histone $\mathrm{H} 3$, particularly in $\mathrm{N}$-terminal tail [9].

DNA regions with homology to histone $H 3$ genes have been found in the genomes of primitive organisms, such as unicellular algae, and $H 3$ gene families have expanded and diversified across plant evolution to reach 14-15 genes in land plants, such as maize and rice [10]. Arabidopsis contains 15 histone $H 3$ genes, including five $H 3.1$ genes, one $H 3.1$-like gene, three $H 3.3$ genes, five $H 3.3$ like genes and one $C E N H 3$ gene [11]. Introns have been found only in the H3.3 genes, three in switchgrass (Rapicum virgatum L.) [12], but not in canonical histone $H 3$ (H3.1), suggesting that the histone variant $H 3.1$ is likely to have evolved from $H 3.3$ [13].

The first plants to have CENH3 identified in were Arabidopsis thaliana [14], maize (Zea mays) [15] and rice (Oryza sativa) [16]. Each of these species has one copy of CENH3, although WGD (whole-genome duplication) has been suggested in all three species. A comparison of the structure of the CENH3 and the canonical histone $\mathrm{H} 3$ proteins showed that they share a conserved histone fold domain at their carboxyl ends [9]. The N-terminal tails of CENH3s, however, differ from those of canonical histone H3s and vary in both length and sequence among different species. As more and more species with monocentric chromosomes (that is, those that have only one centromere) have become known, it comes to the researchers' attention that although most of these species have one copy of $C E N H 3$, some have two or more, which synthesize different variants of proteins. CENH3 duplication in diploid plants was first detected as having occurred in Arabidopsis species, A. halleri and A. lyrata, after their ancestor split from that of $A$. thaliana [17]. Both copies are functional, both have identical exonintron structures (9 exons), but one of them has a 16-bp deletion relative to the original gene in A. thaliana. Subsequently, CENH3 duplications were shown for diploid plants in different taxa: Brassica [18], Pisum and Lathyrus [19], Mimulus [20], Vigna [21]. Both copies produce functional CENH3 proteins, which fully colocalize with each other.

The discovery of $C E N H 3$ duplications complicated the understanding of the evolution of the CENH3 genes and raised the question about the mechanisms and causes of gains, losses and selective constraints. An intriguing aspect of $C E N H 3$ evolution is that the duplications that have occurred in A. halleri, A. lyrata, and Pisum and Lathyrus species are preserved and expressed, while the closely related A. thaliana or Vicia and Lens species, which are in the same tribe Fabeae as are Pisum and Lathyrus, express only a single copy of this central component of centromere specification and function. This motivated us to extend the analysis of CENH3 duplications to other plant taxa. To start with, we chose the cereal family Poaceae. This is one of the largest and most diverse angiosperm families, consisting of more than 12,000 species and more than 700 genera [22]. Most of the economically important species that have long been used in breeding, with numerous crosses involving closely related and distant species, are in this family. The phylogenetic classifications of this family, which were in the past largely based on morphology and anatomy, have in the last three decades been revised based on molecular evidence. Another advantage of Poaceae as an object for studying the evolutionary history of the two-component system of paralogous genes encoding the centromerespecific histone CENH3 is that this family includes not only rice (Oryza sativa) and maize (Zea mays), which have one copy of $C E N H 3$, but also Triticeae species (barley, wheat and rye, to name a few), which have two copies of this gene [23-25].

An increased number of cereal genomes sequenced in recent years and advances in their assembly allowed us to use a homology-based approach and to finally trace back the mutual evolution of the structure of the $\alpha C E N H 3$ and $\beta C E N H 3$ genes and structure of the nearby regions in various tribes in the family Poaceae. We established that around 50 Mya the common ancestor of the subfamilies Bambusoideae, Oryzoideae and Pooideae developed the CENH3 locus composed of the syntenic genes 
CDPK2, CENH3 and bZIP. The duplication event took place within the CENH3 locus in Stipeae and Brachypodieae species around 35-40 Mya. It was accompanied by substantial changes in the exon-intron structure of the daughter gene, $\beta C E N H 3$. The $\alpha C E N H 3$ and $\beta C E N H 3$ genes were subject to different degrees of selection constraints. Purifying selection acts mostly on $\alpha C E N H 3$ s. A lower pressure from purifying selection allowed profound changes to occur in nucleotide/amino acid sequences, especially in the NTT domain of $\beta C E N H 3 s$, in which clade-specific amino acid motifs are present. The other process is that the CENH3 locus is expanding as the distance between $\alpha C E N H 3$ and $\beta C E N H 3$ increases due to a massive insertion of elements of the LTR-containing retrotransposon superfamilies, gypsy and copia. Changes in the molecular structure of the CENH3 locus and the observed general tendency towards its expansion reveal an amazing diversity of ways in which different species implement this scenario.

\section{Results}

\section{Selection of cereal species and identification of CENH3} paralogs in their genomes

Our choice of species was based on the worldwide phylogenetic classification of the family Poaceae, which was created using known molecular markers and morphological characteristics [22]. This classification put together the latest molecular advances with data from a large number of previous morphological studies and placed 771 genera of this family in 51 tribes and 12 subfamilies
[22]. The well-documented presence of one CENH3 variant in maize and rice $[15,16]$ and of two variants in Triticeae [23-25] suggests that CENH3 duplication occurred after the subfamily Pooideae, which includes Triticeae, split from a common ancestor with the subfamily Oryzoideae, which includes rice (Fig. 1). According to Soreng et al. [22], the subfamily between these two is Bambusoideae. Based on this, we identified a selection of promising candidate Poaceae species that could be helpful in tracing the evolutionary history of CENH3 duplication. The list and some details of these species are shown in Table 1, where species belonging to different tribes and subfamilies are arranged in the order of divergefnce according to the classification system we are using [22]. Streptochaeta angustifolia in the subfamily Anomochlooideae was used as an outgroup. Another important factor for choosing candidate species was the availability of high-quality well-annotated genomes. Triticeae species are the most represented in the selection, first, because they have CENH3 paralogs and, secondly, because their genomes are well studied. The study considers all CENH3 genes and CENH3 proteins in the species that are considered to be the progenitors of three ancestral diploid genomes (ABD) of hexaploid wheat (Triticum aestivum)-Triticum urartu (the ancestor of the A genome), Aegilops speltoides (a likely ancestor of the B genome) and Aegilops tauschii (the ancestor of the D genome)-and the ABD subgenomes of T. aestivum. Additionally, the study included two copies of $\alpha C E N H 3$ present in the $\mathrm{N}$ and $\mathrm{K}$ genomes of tetraploid switchgrass

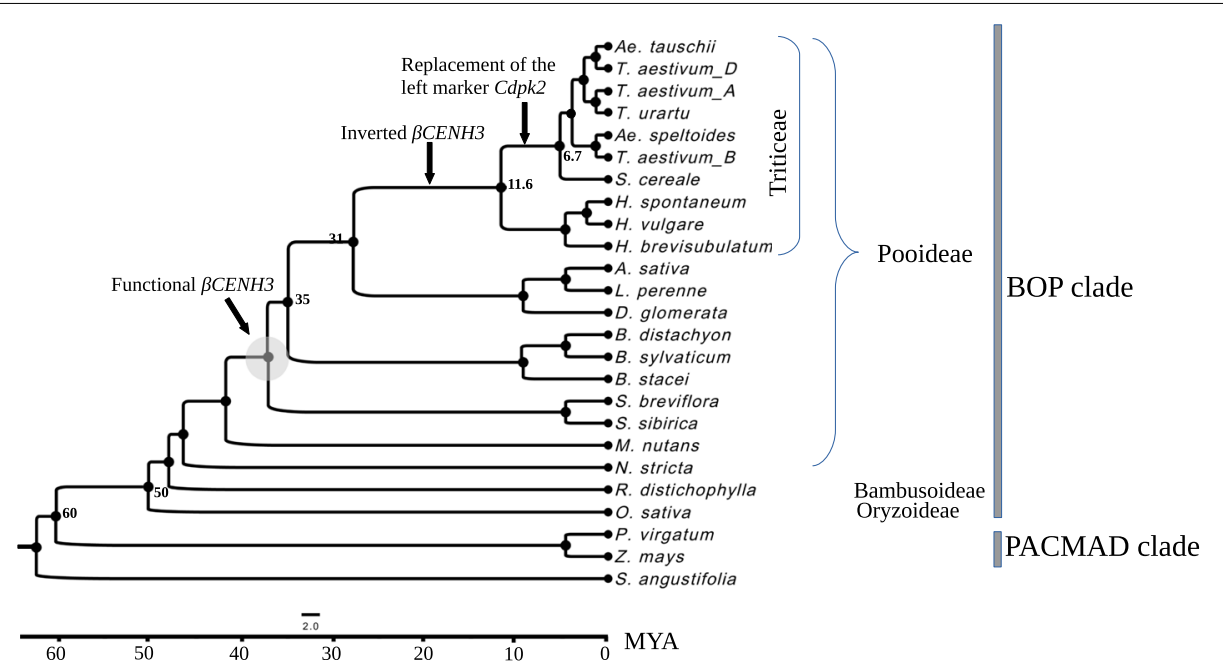

Fig. 1 The phylogenetic tree inferred for the study species according to the classification system of Soreng et al. [22]. Under this system, BOP and PACMAD are two main clades: BOP comprises the subfamilies Bambusoideae, Oryzoideae and Pooideae, and PACMAD comprises the subfamilies Panicoideae, Aristidoideae, Chloridoideae, Micrairoideae, Arundinoideae and Danthonioideae. The subfamilial and tribal attribution of the study species and some of their details are given in Table 1. On the scale: the BOP/PACMAD split time (60 Mya) and various lineage split times according to Chalupska et al. [27] 
Table 1 The study species and their details

\begin{tabular}{|c|c|c|c|c|}
\hline Species & Tribe* & Subfamily* & $\begin{array}{l}\text { Genome size (1C), } \\
\text { Mbp, pg }\end{array}$ & $\begin{array}{l}\text { Ploidy, } \\
\text { chromosome number }\end{array}$ \\
\hline Streptochaeta angustifolia & Streptochaeteae & Anomochlooideae & N/A & $2 n=2 x=22^{* *}$ \\
\hline Panicum virgatum & Paniceae & Panicoideae & $1370^{1} ; 1.4$ & $2 n=4 x=36$ \\
\hline Zea mays & Andropogoneae & Panocoideae & $3280 ; 2.7$ & $2 n=2 x=20$ \\
\hline Oryza sativa & Oryzeae & Oryzoideae & $466 ; 0.5$ & $2 n=2 x=24$ \\
\hline Raddia distichophylla & Olyreae & Bambusoideae & $600^{2} ; \mathrm{N} / \mathrm{A}$ & $2 n=2 x=22^{* * *}$ \\
\hline Nardus stricta & Nardeae & Pooideae & $2050^{1} ; 2.1$ & $2 n=2 x=26$ \\
\hline Melica nutans & Meliceae & Pooideae & $2450^{1} ; 2.5$ & $2 n=2 x=18$ \\
\hline $\begin{array}{l}\text { Stipa sibirica } \\
\text { Stipa breviflora }\end{array}$ & Stipeae & Pooideae & $\sim 980^{1} ; \sim 1.0$ & $2 n=2 x=22$ or 24 \\
\hline $\begin{array}{l}\text { Brachypodium distachyon } \\
\text { Brachypodium sylvaticum } \\
\text { Brachypodium stacei }\end{array}$ & Brachypodieae & Pooideae & $\begin{array}{l}272 ; 0.32 \\
529 ; 0.44 \\
234 ; 0.28\end{array}$ & $\begin{array}{l}2 n=2 x=10 \\
2 n=2 x=18 \\
2 n=2 x=20\end{array}$ \\
\hline Avena sativa & Poeae & Pooideae & $12520^{1} ; 12.8$ & $2 n=6 x=42$ \\
\hline Dactylis glomerata & Poeae & Pooideae & $4420 ; 3.3$ & $2 n=4 x=28^{* *}$ \\
\hline Lolium perenne & Poeae & Pooideae & $2770 ; 2.7$ & $2 n=2 x=14$ \\
\hline Hordeum brevisubulatum & Triticeae & Pooideae & $8800^{1} ; 9.0$ & $2 n=4 x=28$ \\
\hline Hordeum vulgare, ssp. spontaneum & Triticeae & Poideae & $5180^{1} ; 5.3$ & $2 n=2 x=14$ \\
\hline Hordeum vulgare & Triticeae & Pooideae & $5180 ; 5.3$ & $2 n=2 x=14$ \\
\hline Secale cereale & Triticeae & Pooideae & $8460 ; 8.7$ & $2 n=2 x=14$ \\
\hline Triticum urartu & Triticeae & Pooideae & $5.75 ; 5.9$ & $2 n=2 x=14$ \\
\hline Aegilops speltoides & Triticeae & Pooideae & $5680 ; 5.8$ & $2 n=2 x=14$ \\
\hline Aegilops tauschii & Triticeae & Pooideae & $5050 ; 5.2$ & $2 n=2 x=14$ \\
\hline Triticum aestivum & Triticeae & Pooideae & 17,$280 ; 17.7$ & $2 n=6 x=42$ \\
\hline
\end{tabular}

Note: Genome sizes are taken mainly from Leitch et al. [27]. Plant DNA C-values Database (release 7.1). https://cvalues.science.kew.org/

${ }^{1}$ Due to the absence of these values in Databases, they are calculated using the formula: $1 \mathrm{pg}=978 \mathrm{Mbp}$ [28]

2 Li et al. [29]

* tribal and subfamilial attribution to the classification system of Soreng et al. [22]; ** Pohl, Davidse [30]; *** Hunziker et al. [31]

(Panicum virgatum) and two copies of $\beta C E N H 3$ found in oats (Avena sativa). Oats is a hexaploid species, and two copies of $\beta C E N H 3$ ( $\beta C E N H 3 \_1$ and $\left.\beta C E N H 3 \_2\right)$ are localized in different subgenomes (Avena sativa - OT3098 v2, PepsiCo, https://wheat.pw.usda.gov/jb? data $=$ /ggds/oat-ot3098v2-pepsico). To identify genomic copies of $C E N H 3$, we used the fully sequenced genomes or extended contigs of promising candidate species and transcriptomes available in the NCBI and Phytozome databases (Table 1 and Additional file 1: Table S1). To make use of the partially assembled genomes of Stipa sibirica and Lolium perenne, we extracted available reads from the SRA at NCBI (https://www.ncbi.nlm.nih.gov) and assembled them into contigs using Minia [26].

Two considerations are important for further reading. One relates to the analysis of the genome of cultivated barley (Hordeum vulgare L.). According to the most updated version of its genome, GCA_902498975.1, $\beta C E N H 3$ is on chromosome $1 \mathrm{H} 72 \mathrm{~kb}$ away from $\alpha C E N H 3$. Unlike the $\beta C E N H 3$ s in other Triticeae species, the one in question contains a 18-bp deletion in the NTT and a stop codon in exon 3 in the $3^{\prime}$-end of the HFD and, therefore, the protein it synthesizes should be as short as 126 amino acids (aa), which is 28 aa shorter than its ortholog in wild barley Hordeum brevisubulatum (Additional file 2: Fig. S1). The structure of $\beta C E N H 3$ in Hordeum vulgare subsp. spontaneum (hereinafter H. spontaneum), which is the closest relative and progenitor of cultivated barley [33], is similar to that of its ortholog in $H$. vulgare. Because it has been shown that the C-terminal tail of CENH3 is responsible for interactions between $\mathrm{CENH} 3$ and histone $\mathrm{H} 4$ in nucleosomes and the last five amino acids in the $\mathrm{C}$-terminus are necessary for CENH3 deposition [34], it is unlikely that a protein molecule so shortened will be functional. One more copy of $\beta C E N H 3$ was found on barley chromosome $6 \mathrm{H}$, with no alpha paralog around. This copy has a 17 -aa deletion in the $\mathrm{N}$-terminus, the deletion being longer than that in $1 \mathrm{H}$, and a normalsize C-terminus (Additional file 2: Fig. S1). Because antibodies against $\beta$ CENH3 had previously been 
obtained and located in barley centromeres by immunostaining [35], it seems logical to assume that this $\beta C E N H 3$ sequence may be capable of producing a functional $\beta C E N H 3$ molecule. In further analysis, we will use both copies of $\beta C E N H 3$ from chromosomes $1 \mathrm{H}$ and $6 \mathrm{H}$, and the CENH3 paralogs from H. brevisubulatum.

The other consideration relates to difficulties in identifying the functional $\beta C E N H 3$ transcripts in Brachypodium distachyon. The genome of this species is small and compact, is often considered a model for the large and often polyploid genomes of Triticeae species and is well studied [36, 37]. It was therefore important to find out when exactly CENH3 duplication occurred in Pooideae: before or after the speciation of Brachypodium. It had been previously argued that only one CENH3 paralog is present in $B$. distachyon [38, 39], $1776 \mathrm{bp}$ in size, which is substantially larger than $\alpha C E N H 3$ in the other Triticeae species. We extracted the genomic sequences of $C E N H 3$ with adjacent regions for $B$. distachyon from GenBank, and for two other species, Brachypodium stacei and Brachypodium sylvaticum, from the Phytozome database. The sequence of the 1776-bp transcript, LOC100830307, is annotated in the genome of $B$. distachyon (NC_0161323) (Additional file 2: Fig. S2). A comparison with known sequences from other cereal species revealed that this transcript has homologies with both $\alpha C E N H 3$ and $\beta C E N H 3$. However, its exon 2 has a stop codon in it. Similar degenerate copies or truncated fragments of $C E N H 3$ have been found on two soybean chromosomes (Glycine max) [19].

We wanted to find out whether or not the stop codon in exon 2 of the $\beta C E N H 3$ gene in $B$. distachyon from GenBank was a sequencing error. To do so, we obtained cDNA from RNA, line B21 and resequenced the transcripts from locus LOC100830307. All versions of the transcripts and the primers to the $5^{\prime}$ - and $3^{\prime}$-ends of $\beta C E N H 3$ and $\alpha C E N H 3$ are schematically shown in Additional file 2: Fig. S3. The vast majority of $\beta C E N H 3$ transcripts (92\%) had a stop codon at the same position in them as in genome sequence NC_0161323. It is therefore obvious that the annotated $B$. distachyon genome contains both CENH3 paralogs.

An aberrant beta form (Bstacei_316_v1.0, Phytozome) with stop codons was also found in B. stacei (Additional file 2: Fig. S2). Additionally, this species has the full-length beta form Brast08G079400.1.p, but its HFD sequence is substantially different from its counterparts in other species. In B. sylvaticum, no transcript of either CENH3 paralog has a stop codon; here Brasy8G088800.1.p is given as an example (Additional file 2: Fig. S2). Thus, among the three Brachypodium species studied, only $B$. sylvaticum has been found to have $\beta C E N H 3$ that is capable of producing a functional protein molecule. $\beta C E N H 3$ of this species was used in further analysis.

\section{CENH3 protein sequence alignments and phylogenetic trees}

To identify the genomic copies of CENH3, we ran sequence similarity searches in the fully sequenced genomes of promising candidate species and transcriptomes from NCBI and Phytozome databases (Additional file 1: Table S1), and the CENH3-containing genomic loci assembled by us for Stipa sibirica and Lolium perenne (Additional files 3 and 4). From the species for which only transcriptomes are known (Stipa breviflora, Melica nutans and Nardus stricta), only cDNA and protein sequences were collected and analyzed (Additional files $5,6,7,8)$. The nucleotide or amino acid sequence of $C E N H 3$ in a particular species or in its closest relatives was used as a search query. It was found that the transcriptomes of N. stricta (the tribe Nardeae) and $M$. nutans (the tribe Meliceae) have only $\alpha C E N H 3$, while Stipeae species and the species in the other Pooideae tribes (Brachypodieae, Poeae and Triticeae) have two, $\alpha C E N H 3$ and $\beta C E N H 3$. Thus, according to the classification system of Soreng et al. [22], two functional CENH3 paralogs appeared in Pooideae either before Stipeae and Brachypodieae split or independently thereafter (Fig. 1).

Multiple alignments of the deduced amino acid sequences of the $\alpha \mathrm{CENH} 3$ and $\beta C E N H 3$ proteins found in the species listed in Tables 1, 45 samples in total, were obtained using the MUSCLE algorithm [40] (Fig. 2) and Bali-Phy [41] (Additional file 2: Fig. S4). Phylogenetic trees were inferred using the Maximum Likelihood (ML) method and JTT matrix-based model [42]. Evolutionary analyses were conducted in MEGA X [43]. The tree constructed using MUSCLE with the highest log likelihood $(-4953.02)$ is shown in Fig. 3. The trees constructed using these alignment methods show (1) a good discrimination between the species that have only one form of CENH3 (alpha) and the species that have two forms of CENH3 (alpha and beta) and (2) a good discrimination between the $\alpha \mathrm{CENH} 3$-only clade (in a gray-filled shape) and the $\beta C E N H 3$-only clade (in a light-blue-filled shape). The 'core' Pooideae tribes Triticeae and Poeae [44] form distinct clusters within both clades, consistent with their positions in the classification system, which is based on the largest number of different characters [22]. Of special interest is the position of CENH3 proteins in S. sibirica, S. breviflora and B. sylvaticum, because these were the first species reported to have both CENH3 paralogs at once. On the ML tree, (Fig. 3) aCENH3s of Stipa species cluster is well separated from the cluster with $\alpha \mathrm{CENH} 3 \mathrm{~s}$ of Brachypodium species. Looking at this topology, we can readily suggest that the probable scenario is that of an 


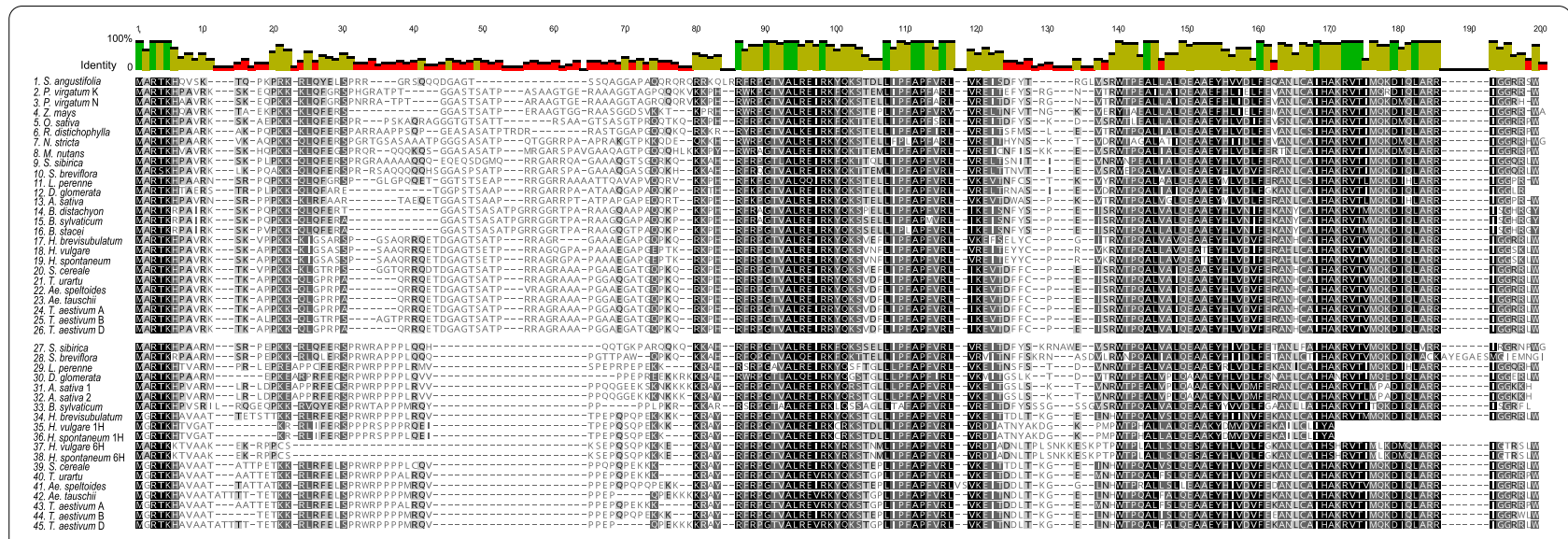

Fig. 2 A multiple alignment of the amino acid sequences of CENH3 proteins using the MUSCLE program [40]

For convenience, the alpha and beta forms are grouped into two separate blocks: 1-26 (aCENH3) and 27-45 ( $\beta C E N H 3)$. Amino acid residues identical in all species are shown as white letters on the black background.

independent duplication of CENH3 in Stipa and Brachypodium species. The cluster with the $\beta$ CENH3 copies of the Stipa species and B. sylvaticum (in a dark-blue-filled shape) lies separately, closer to the alpha-only clade. This intermediate position of the beta forms in these species suggests the presence of specific features-motifs-in their structure, characteristic of the alpha form of the protein.

In all species, the N-domains of both CENH3 paralogs have a more heterogeneous structure than do the $\mathrm{C}$-termini. That becomes especially evident when amino acid alignments are generated with Bali-Phy (Additional file 2: Fig. S4). Specific motifs (most in the NTT) characteristic of certain groups of species are contained in the rectangular boxes with different border colors. In S. sibirica and S. breviflora, the NTTs of $\beta$ CENH3 have 10 and 9 amino acid residues (red borders), respectively, that are present only in the alpha forms and are not present in the beta forms in other species except the Stipa species. The HFDs of $\beta$ CENH3 in the Stipa species also have species-specific motifs in the loop1 region. The $\beta C E N H 3$ of the Pooideae species with both paralogs have specific
NTT motifs characteristic of Triticeae and Poeae (green and pink borders, respectively). Barley stands out from all the other Triticeae species - this can be seen from the topology of the phylogenetic trees: it forms a cluster well separated from other species (Fig. 3) and has a species-specific amino acid motif in the loop1 region of the $\beta C E N H 3$ HFD (Additional file 2: Fig. S4). Noteworthy, both paralogs of wild barley $H$. brevisubulatum are closer to other Triticeae species than are those of $H$. vulgare.

\section{Lineage-specific evolution}

Gene duplication generally implies the reproduction of the sequence of a gene in its copy. It seems logical to assume that differences in the structure of copies will characterize the rates of their evolutionary changes and the degree of their divergence since the duplication event. One of the quantitative characteristics of these differences may be the degree of homology of the amino acid and/or nucleotide sequences. A comparison of the amino acid sequences of $\alpha \mathrm{CENH} 3$ and $\beta \mathrm{CENH} 3$ aligned using FASTA showed that their similarity ranges from 65 to $70 \%$ in most species (Table 2). This is substantially lower

\footnotetext{
(See figure on next page.)

Fig. 3 The phylogenetic tree inferred from a comparison of the deduced CENH3 proteins using the ML method, the JTT matrix-based model (Jones et al. 1992) and bootstrapping (1000 replicates). Initial tree(s) for the heuristic search were obtained automatically by applying Neighbor-Join and BioNJ programs to a matrix of pairwise distances estimated using a JTT model, and then selecting the topology with superior log likelihood value. The tree with the highest log likelihood (-4953.06) is shown. A discrete Gamma distribution was used to model evolutionary rate differences among sites ( 5 categories $(+G$, parameter $=1.0416)$ ). The tree is drawn to scale, with branch lengths measured in the number of substitutions per site. This analysis involved 45 amino acid sequences. There were a total of 200 positions in the final dataset. Evolutionary analyses were conducted in MEGA X [43]. Bootstrap values were calculated from at least 1000 replications. Amino acid sequences were aligned using MUSCLE with default settings. On the branches: bootstrap values. The aCENH3-only clade is in a gray-filled shape, the $\beta C E N H 3$-only clade is in a light-blue-filled shape; the $\beta C E N H 3$ clade comprised of Stipa spp. and Brachypodium sylvaticum is in a dark-blue-filled shape
} 


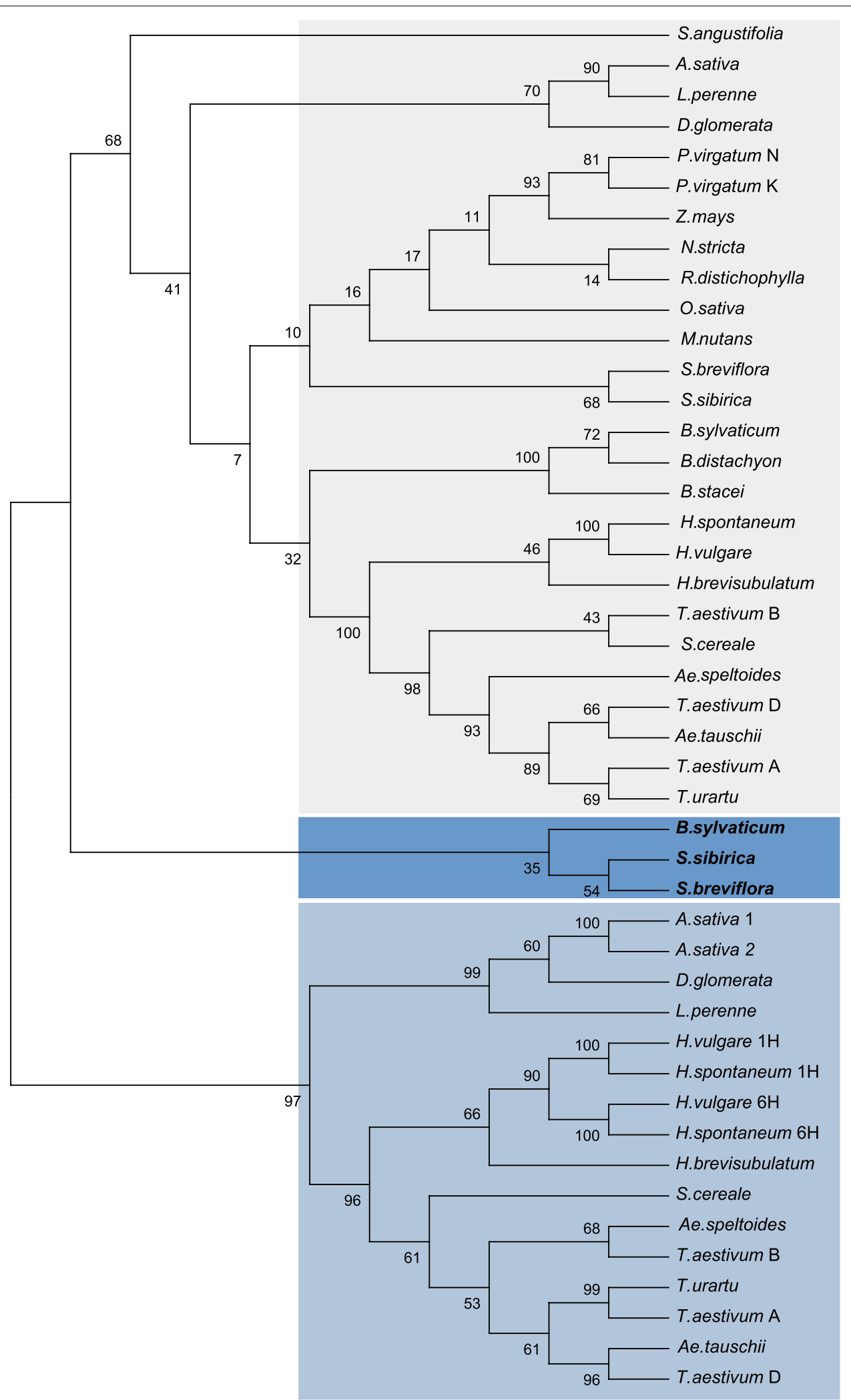

Fig. 3 (See legend on previous page.) 
Table 2 The similarity of the deduced amino acid sequences of aCENH3 and $\beta C E N H 3$

\begin{tabular}{|c|c|c|c|c|}
\hline Species & Tribe & $\begin{array}{l}\text { Similarity of protein copies, } \\
\%\end{array}$ & Similarity of NTTs, $\%$ & $\begin{array}{l}\text { Similarity } \\
\text { of HFDs, } \%\end{array}$ \\
\hline $\begin{array}{l}\text { Stipa sibirica } \\
\text { Stipa breviflora }\end{array}$ & Stipeae & $\begin{array}{l}77.5 \\
75.2\end{array}$ & $\begin{array}{l}64.9 \\
58.4\end{array}$ & $\begin{array}{l}87.5 \\
89.8\end{array}$ \\
\hline Brachypodium sylvaticum & Brachypodieae & 70.4 & 52.9 & 83.5 \\
\hline Lolium perenne & Poeae & 69.0 & 42.7 & 90.2 \\
\hline Dactylis glomerata & Poeae & 71.7 & 50.7 & 87.8 \\
\hline $\begin{array}{l}\text { Avena sativa } \beta 1^{*} \\
\beta 2\end{array}$ & Poeae & $\begin{array}{l}65.7 \\
66.1\end{array}$ & $\begin{array}{l}42.7 \\
55.6\end{array}$ & $\begin{array}{l}86.7 \\
86.7\end{array}$ \\
\hline Hordeum brevisubulatum & Triticeae & 74.7 & 53.4 & 91.4 \\
\hline $\begin{array}{l}\text { Hordeum spontaneum } 1 \mathrm{H}^{* *} \\
6 \mathrm{H}\end{array}$ & Triticeae & $\begin{array}{l}62.9 \\
65.5\end{array}$ & $\begin{array}{l}45.6 \\
42.7\end{array}$ & $\begin{array}{l}81.9 \\
82.8\end{array}$ \\
\hline $\begin{array}{l}\text { Hordeum vulgare } 1 \mathrm{H}^{* *} \\
6 \mathrm{H}\end{array}$ & Triticeae & $\begin{array}{l}61.4 \\
64.9\end{array}$ & $\begin{array}{l}54.0 \\
42.7\end{array}$ & $\begin{array}{l}81.9 \\
82.8\end{array}$ \\
\hline Secale cereale & Triticeae & 70.4 & 44.9 & 93.5 \\
\hline Triticum urartu & Triticeae & 69.6 & 45.8 & 90.3 \\
\hline Aegilops speltoides & Triticeae & 67.8 & 45.0 & 88.4 \\
\hline Aegilops tauschii & Triticeae & 70.4 & 43.4 & 91.4 \\
\hline $\begin{array}{l}\text { Triticum aestivum } A^{* * *} \\
\text { B } \\
\text { D }\end{array}$ & Triticeae & $\begin{array}{l}69.6 \\
71.3 \\
70.4\end{array}$ & $\begin{array}{l}43.4 \\
44.3 \\
43.4\end{array}$ & $\begin{array}{l}90.3 \\
92.5 \\
91.4\end{array}$ \\
\hline
\end{tabular}

Note: Separate HFD regions are singled out according to Karimi-Ashtiyani et al. [45]

* data for two copies of $\beta C E N H 3$ in Avena sativa

** data for $\beta C E N H 3$ on chromosomes $1 \mathrm{H}$ and $6 \mathrm{H}$

** data for the A, B and D genomes of Triticum aestivum

than the $75 \%$ similarity between the two paralogous proteins, CenH3-1 and CenH3-2, in Fabeae species [19] and $91 \%$ similarity between the proteins CENH3.1 and CENH3.2 in Vigna unguiculata [21]. However, S. sibirica and $S$. breviflora have markedly higher similarity values relative to other species, 77.5 and $75.2 \%$, respectively. The difference in similarity between Stipa and other species is stronger in the NTTs than in the HFDs (Table 2). Thus, either the rate of divergence of the CENH3 paralogs was lower in Stipa species than in most Pooideae species that have two CENH3 paralogs or duplications in Stipa are more recent events.

The type of selective pressure acting on the CENH3 genes during their diversification was identified based on the observed ratios of nonsynonymous (Ka) to synonymous $(\mathrm{Ks})$ nucleotide substitution rates $(\mathrm{Ka} / \mathrm{Ks}=\omega)$ separately for each ortholog. All-to-all pair-wise comparisons of full-length CENH3 coding sequences using the Ka-Ks calculator [46] estimated $\omega$ to be less than 1 for each of the orthologs in all pairs of species being compared (Additional file 1: Tables S2, S3), indicating purifying (stabilizing) selection as the major force shaping the diversity of these genes. For $\alpha C E N H 3$, this ratio was in all cases substantially lower, with most values falling in the range of 0.2-0.4 (Additional file 1: Table S2), indicating a considerable strong purifying selective pressure.
For $\beta C E N H 3$, the $\omega$ values were higher, in the range of $0.4-0.7$, and for two cases of $\beta C E N H 3$ located on chromosome $6 \mathrm{H}$ of $H$. vulgare they exceeded 1 (highlighted in bold, Additional file 1: Table S3). Although the observed excess fails to reach significance, it is sufficient for concluding that $\beta C E N H 3$ paralogs are under relaxed selective pressure.

The dominating impact of purifying selection does not exclude the possibility that diversifying positive selection acts on specific branches of phylogenetic trees. We extended our analysis so far as to identify specific branches that have sites evolving with $\omega>1$ and used adaptive branch site-random effects likelihood (aBSREL) [47]. This branch-site model simultaneously implements $\omega$ variation across branches and sites. aBSREL requires no prior knowledge about which lineages are more likely to have experienced episodic diversifying selection. Significance was assessed by the likelihood ratio test (LRT) at a threshold of $\mathrm{P} \leq 0.05$. The most statistically significant (at $P<0.05$ ) evidence of episodic diversifying selection is found on the branch leading to the cluster (node 11, $P=0.0073$ ) containing $\beta C E N H 3$ in S. sibirica, S. breviflora and $B$. sylvaticum (Additional file 2: Fig. S5b). No diversifying selection was noted in the cluster with the Triticeae species, with the exception of the branch with $\beta C E N H 3$ 


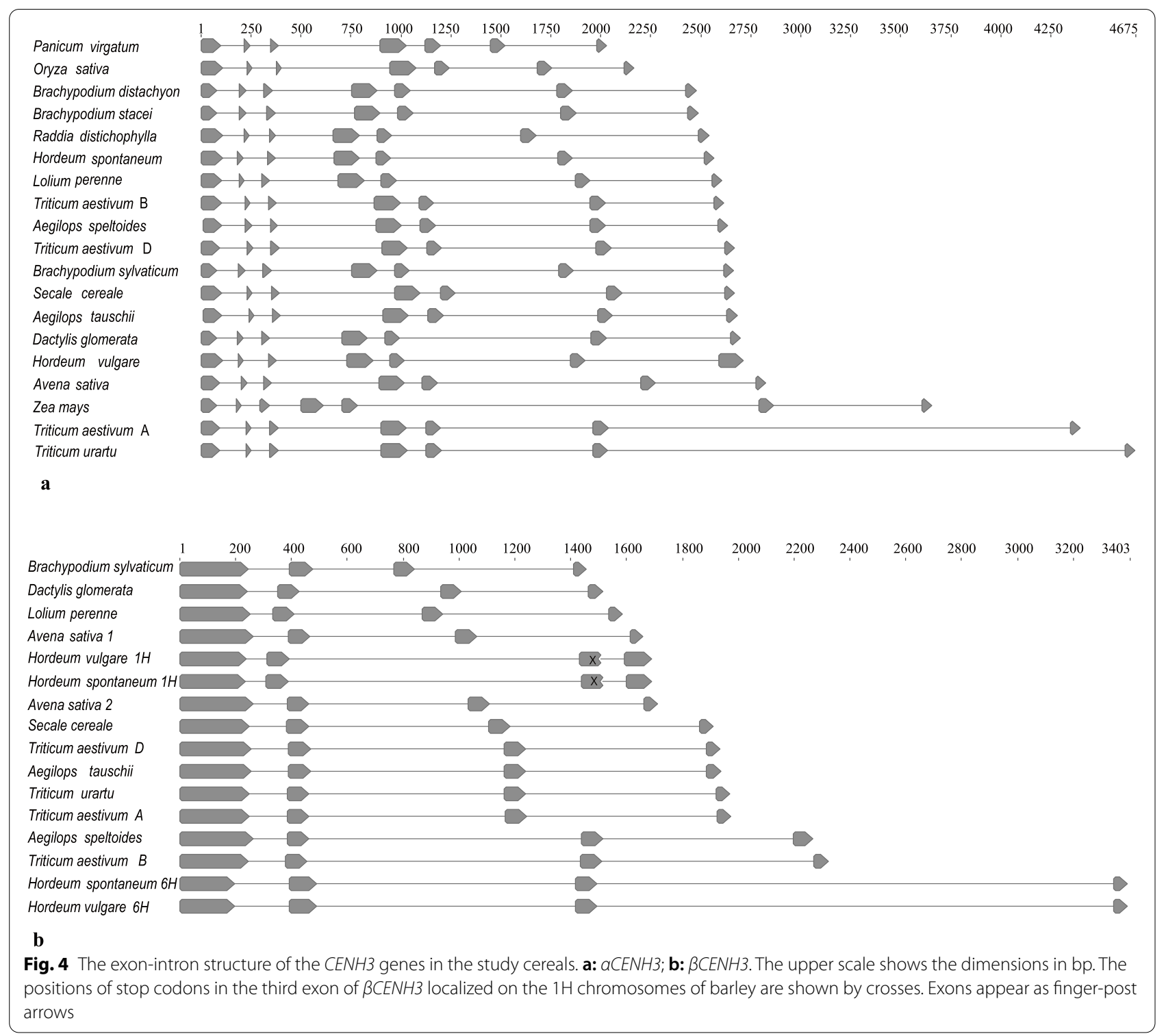

on chromosome $6 \mathrm{H}$ of $H$. vulgare $(P=0.0447)$. The percentage of the sites driven by positive selection in each case is indicated in brackets in Additional file 2: Fig. S5.

In the tree constructed for $\alpha \mathrm{CENH} 3$ with BSREL (Additional file 2: Fig. S5a), B. sylvaticum is clustered with the other Brachypodium species and the greatest influence of diversifying selection is exerted on the branches leading to the cluster of Stipa species (node $34, P=0.0448)$ and further to $S$. sibirica $(P=0.0000)$. Thus, a good match can be observed on the branches, in which some of the sites within the alpha and beta forms of the centromere-specific histone undergo positive diversifying selection.
The exon-intron structure of $\alpha C E N H 3$ and $\beta C E N H 3$

Transcriptome libraries were used to identify $C E N H 3$ transcripts (Additional files 5-8) and to infer the exonintron structure of $\alpha C E N H 3$ and $\beta C E N H 3$ in those cereal species in Table 1 for which both genomic and transcriptome libraries were available. A comparison of alpha orthologs showed that the $\alpha C E N H 3$ structure is conserved, no matter whether a given species has both paralogs or only the alpha. Each $\alpha C E N H 3$ gene has seven exons encoding proteins 159-172 amino acids in length and separated by six introns (Fig. 4a). The total size of the $\alpha C E N H 3$ genes varies considerably across species, from 2050 to $4679 \mathrm{bp}$, due to differences in intron sequences. The introns at the $3^{\prime}$-end of $\alpha C E N H 3$ are much longer 


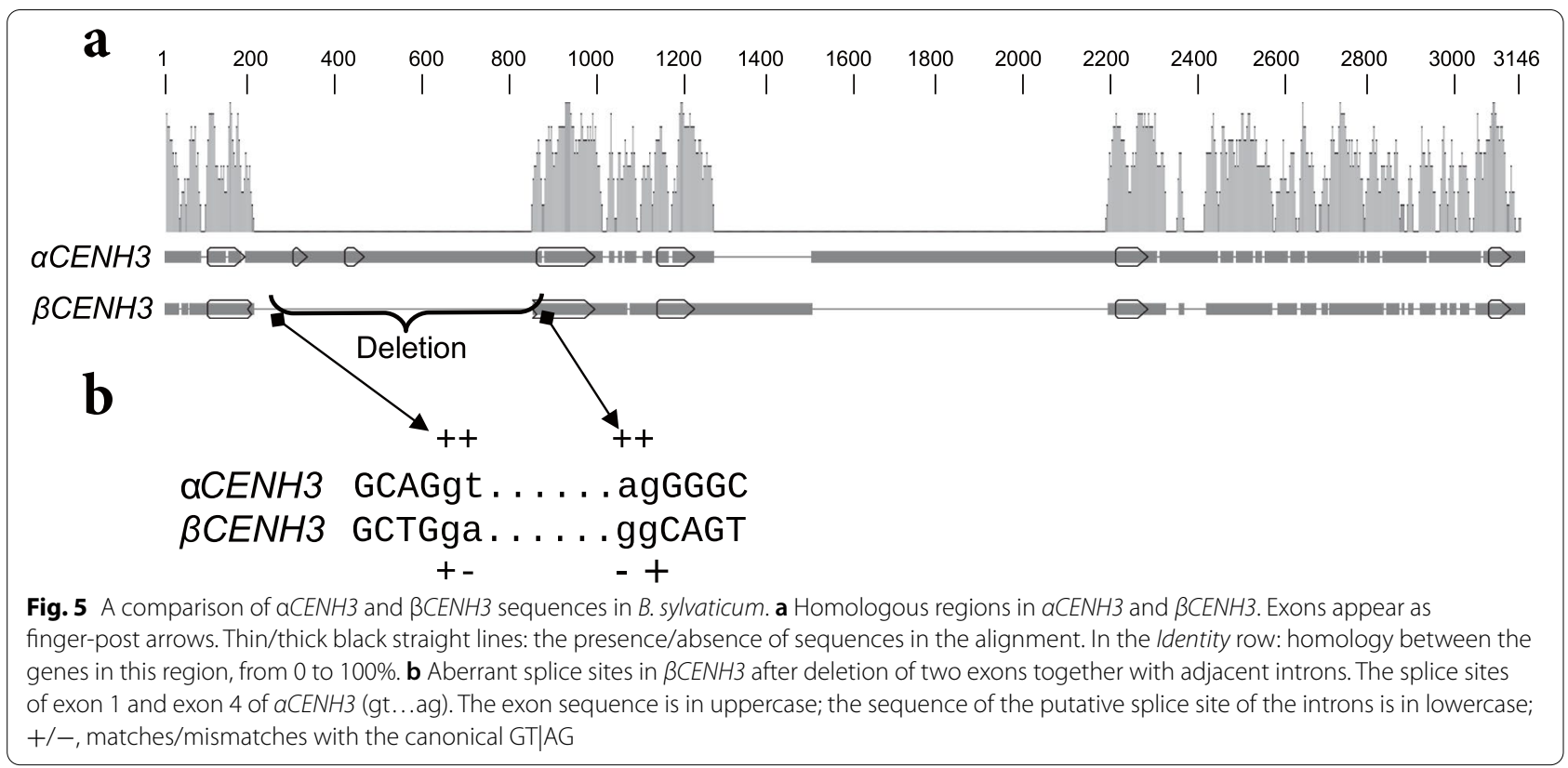

than those at its $5^{\prime}$-end, and introns 6 of $\alpha C E N H 3$ in $T$. urartu and the A genome of T. aestivum are the longest, 2588 and $2312 \mathrm{bp}$, respectively.

The structural features of the $\alpha C E N H 3$ paralogs - a conserved number of exons and introns in different species and longer introns at the $3^{\prime}$-ends of the genes-are also typical of all $\beta C E N H 3$ paralogs. However, the latter contain fewer exons and introns: four exons and three introns. An exon-intron structure similarly different from the $\alpha C E N H 3$ genes is found in Brachypodium, one of the first tribes to have had these two paralogs. It is logical to assume that these changes have nothing to do with the post-duplication evolutionary history, but accompanied the duplication event.

As follows from the results presented, two functional paralogs of CENH3 first appeared in Stipeae and Brachypodium. We carried out a detailed comparative analysis of the genomic copies in B. sylvaticum. The sequences of the $\alpha C E N H 3$ and $\beta C E N H 3$ genes together with the adjacent $5^{\prime}$ - and $3^{\prime}$ - regions at the CENH3 locus were analyzed and compared using MUSCLE (Fig. 5). As can be seen, the shaping of the $\beta C E N H 3$ structure was accompanied by an extended deletion including the complete sequences of exon 2 and exon 3 and a part of the $3^{\prime}$-end of exon 1 and of the $5^{\prime}$-end of exon 4, together with the corresponding introns (Fig. 5a). Interestingly, the $3^{\prime}$-boundary of the resulting extended exon-exon $1-$ in $\beta C E N H 3$ matched the $3^{\prime}$-boundary of exon 4 in $\alpha C E N H 3$. Figure $5 \mathrm{~b}$ shows aberrations in the splice sites of $\beta C E N H 3$ after deletion of two exons. The exon-intron structure described is characteristic of $\beta C E N H 3$ in all subsequent species on our evolutionary scale (Fig. 4B). The size of exon 1 of $\beta C E N H 3$ in different species is $223-264 \mathrm{bp}$, which notably exceeds the size of exon 1 of $\alpha C E N H 3$, $76-127$ bp (Fig. 4). Thus, $\beta C E N H 3$ has undergone such profound changes in nucleotide sequences (and, consequently, amino acid sequences) and in exon-intron structure that it is so different from $\alpha C E N H 3$ now.

\section{The structure of the CENH3 locus in evolution}

We examined the genomic environment of the $\alpha C E N H 3$ and $\beta C E N H 3$ paralogs and found that upstream of $\beta C E N H 3$ in most of the studied genomes are the $L H C B-l$ gene, which encodes chlorophyll a-b binding protein $3 \mathrm{C}$, in Triticeae species and the CDPK2 gene, which encodes calcium-dependent protein kinase 2 , in the other species. Downstream of $\alpha C E N H 3$ in all species is a $b Z I P$ transcription factor gene. Thus, these genes, together with $C E N H 3$, can be considered a syntenic group or the CENH3 locus, with CDPK2 and bZIP as its left-hand and right-hand boundary, respectively. We assumed that a detailed analysis of the molecular structure of the CENH3 locus would facilitate our understanding of its evolutionary history. Figure 6a shows a schematic of this locus in some cereal species. In maize, which, according to the classification system of Soreng et al. [22], is in the PACMAD clade (Fig. 1), no CENH3 locus with the left-hand or the right-hand marker gene has been found; the genes closest to $\alpha C E N H 3$ are those for leucine-rich repeat (LRR) family protein and non-specific lipid transfer protein GPI-anchored 2. 


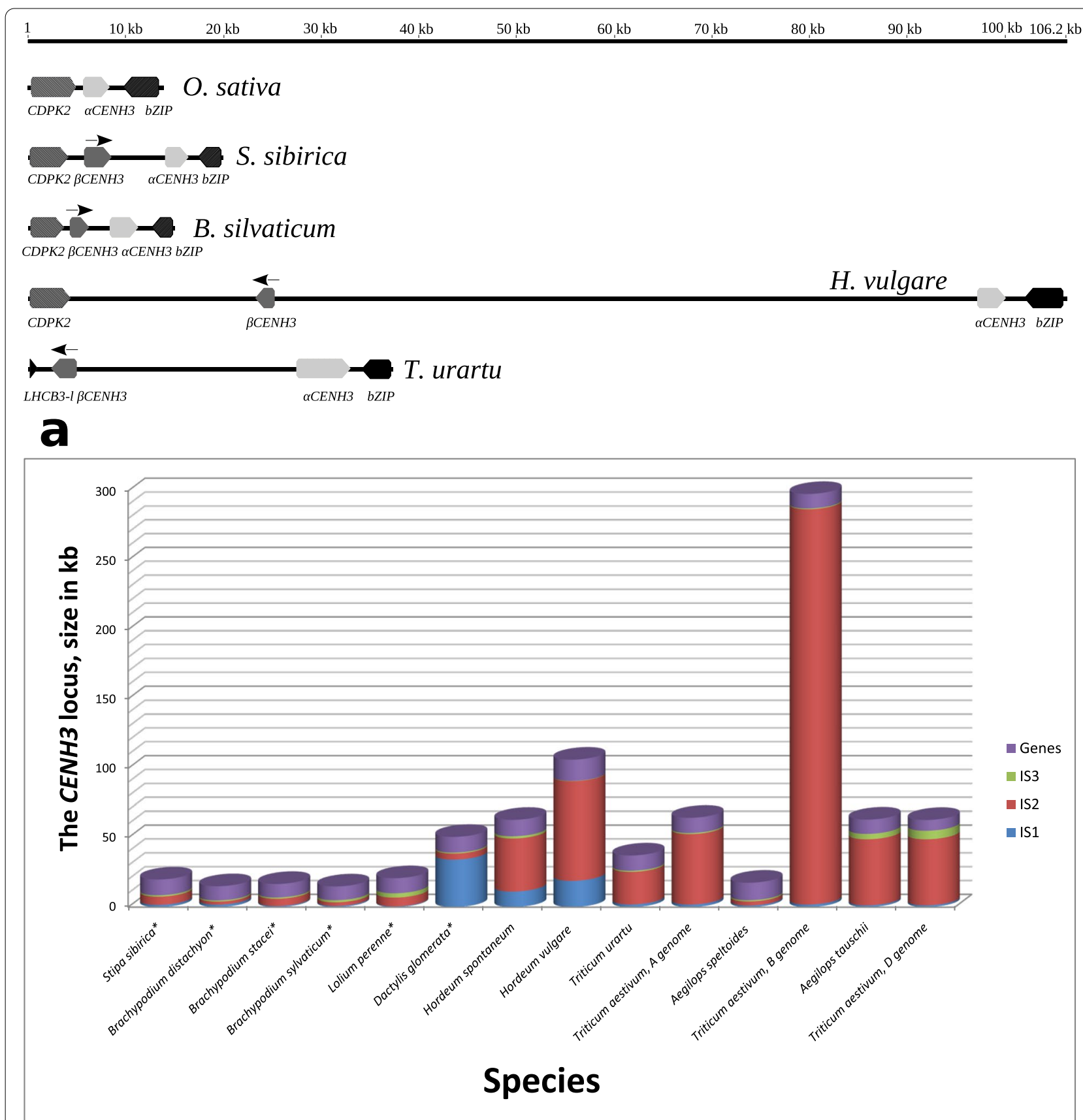

\section{b}

Fig. 6 The structure and evolutionary changes of the CENH3 locus. a Syntenic genes comprising the CENH3 locus. The species with important events, such as the formation of a syntenic group (rice), the emergence of the beta paralog (Stipa, Brachypodium), the inversion of the beta paralog (barley) and replacement of the $5^{\prime}$ marker gene (T. urartu) in their evolutionary history are given as examples. b The evolutionary changes in the size of the genes and intergenic spacers (ISS) comprising the CENH3 locus in Pooideae. Species not belonging to the tribe Triticeae, are marked with asterisks

This classification system we used assigns the Triticeae species, in which two paralogs of the centromeric histone gene were first found (barley, rye and wheat), to the BOP clade, which includes the subfamilies Bambusoideae,
Oryzoideae and Pooideae (Fig. 1). According to the established order of divergence from a common ancestor of the BOP and PACMAD clades, the first species in our set is rice (O. sativa). In the rice genome, the 
above-described collinearity of genes is present and the CENH3 locus is short, $15.7 \mathrm{~kb}$, of which $2.18 \mathrm{~kb}$ is contributed by one copy of CENH3 (Fig. 6a). $R$. distichophylla, a species in the subfamily Bambusoideae, has the CENH3 locus with a single copy of the gene, as does rice.

Two functional paralogs of this gene, $\beta C E N H 3$ and $\alpha C E N H 3$, appear in Stipeae and Brachypodieae, both paralogs being in the same $5^{\prime} \rightarrow 3^{\prime}$ orientation. The size of the locus in $B$. distachyon is very close to that in rice $(15 \mathrm{~kb})$, and $S$. sibirica has it as large as nearly $20 \mathrm{~kb}$. The distances between $\beta C E N H 3$ and $\alpha C E N H 3$ (intergenic spacer, IS2) range from $2.16 \mathrm{~kb}$ in $B$. distachyon to $5.9 \mathrm{~kb}$ S. sibirica (Fig. 6b). In S. sibirica, most of IS2, $3.27 \mathrm{~kb}$, is given to the non-LTR retrotransposon LINE134_SBi. Additionally, the locus reveals a short 215-bp fragment of the transposable element Harbinger and a few relatively short (a few dozens of base pairs) tracks of simple repeats. IS2 between the CENH3 paralogs in Brachypodium species has much the same composition. Curiously, the intergenic spacers in all these species do not have the smallest fragments of LTR-containing retrotransposons, the most common class of transposable elements in plants.

Poeae species are observed to have an increased size of the entire CENH3 locus (Fig. 6b). Not only the already mentioned classes of DNA sequences-LINE, simple repeats and short fragments of various DNA transposon families-contribute considerably to the increase in these sizes, but also extended fragments of LTR retrotransposons. Two main superfamilies of LTR retrotransposons, copia and gypsy, are nearly equal contributors in extended IS2. D. glomerata is found to have families that are also present in the IS2 of the Triticeae species: Eugene, Usier, Inga (all belong to copia), Sabrina, Sabina, Egug, Romani, Fatima (all belong to gypsy). It should be noted that, despite an impressive size of IS2 in these species, not a single LTR retrotransposon family has been found to be present in them as a full-length element: most of them occur as truncated or degenerate copies. This patchwork-like arrangement suggests that IS2 has undergone multiple recombination events throughout evolution and that no transposable element has been inserted in this locus since a recent past.

The available complete versions of sequenced genomes of several Triticeae species make it possible to trace back the evolution of the CENH3 locus in better detail than we can do to other tribes and to assess the contribution of wild progenitor species to the formation of this locus in the corresponding cultivated derivatives. In cultivated barley $H$. vulgare, the CENH3 locus is located on chromosome $1 \mathrm{H}$ and occupies almost $100 \mathrm{~kb}$ (Fig. 6a and b). A characteristic feature of the locus is that $\beta C E N H 3$ is in the opposite direction to $\alpha C E N H 3$, which is indicative of an inversion. As a result of the inversion, $\beta C E N H 3$ and $\alpha C E N H 3$ assume a head-to-head orientation, and this is typical of all other Triticeae species, making them different from Poeae species. Unlike barley, other Triticeae species do not have a stop codon in exon 3 in the 3 '-end of the HFD and, therefore retain the full-length sequence of $\beta C E N H 3$. One more feature of the DNA composition of the CENH3 locus in $H$. vulgare is a high abundance of copia elements in it - they are nearly four times as abundant as gypsy elements (Table 3). We measured their ratio in barley chromosomes $1 \mathrm{H}$ and $6 \mathrm{H}$, each having one copy of $\beta C E N H 3$, and found it to be 2.05:1, which is equal to the value determined for the entire barley genome [48]. Thus, the CENH3 locus in $H$. vulgare has an unusually high concentration of copia elements, substantially higher than their genome-average abundance in this species. The dominant copia family of LTR retrotransposons at the $C E N H 3$ locus, as in the entire $H$. vulgare genome, is $B A R E-1$, with its 7 truncated elements contributing to $19.1 \mathrm{~kb}$ of the locus or $40.6 \%$ of all copia elements at the locus (Additional file 1: Table S4).

Table 3 The percentage abundance of the main DNA classes in the intergenic spacers of the CENH3 locus in Triticeae

\begin{tabular}{|c|c|c|c|c|c|}
\hline Species & $\begin{array}{l}\text { Copia } \\
\text { superfamily }\end{array}$ & $\begin{array}{l}\text { Gypsy } \\
\text { superfamily }\end{array}$ & Gypsy/Copia & Transposons & Others \\
\hline Hordeum spontaneum & 27.2 & 21.4 & 0.79 & 7.9 & 6.4 \\
\hline Hordeum vulgare & 50.9 & 13.0 & 0.25 & 3.0 & 3.4 \\
\hline Triticum urartu & 28.9 & 15.8 & 0.55 & 0 & 14.4 \\
\hline Triticum aestivum, A genome & 19.4 & 57.5 & 2.96 & 1.8 & 7.3 \\
\hline Aegilops speltoides & 0 & 1.5 & & 13.7 & 23.1 \\
\hline Triticum aestivum, B genome & 22.0 & 34.1 & 1.55 & 7.0 & 4.7 \\
\hline Aegilops tauschii & 23.2 & 44.3 & 1.91 & 2.8 & 1.2 \\
\hline Triticum aestivum, D genome & 22.2 & 42.4 & 1.91 & 0.5 & 1.0 \\
\hline
\end{tabular}

NOTE. Note: Only Triticeae species are presented, because only these species, except for Aegilops speltoides, have been identified as having upwards of $60 \%$ DNA sequences in the most common classes and superfamilies of transposable elements. The Others column is for non-LTR retrotransposons, simple repeats and unclassified sequences 
Two other copia families, Maximus and Inga, are represented by fragments: six in the former $(17.7 \mathrm{~kb}$ in total) and four in the latter (10 kb in total). The CENH3 locus is half as long in wild barley $H$. spontaneum as in cultivated barley (Fig. 6b), and the $\beta C E N H 3$ gene, together with its $5^{\prime}$-region, is in an inverted orientation, as in $H$. vulgare. The locus has many more fragments of gypsy elements in $H$. spontaneum than in $H$. vulgare. The composition of retrotransposon families and LINE families in both barley species are identical; however, Maximus is represented in the locus of $H$. spontaneum only by one short fragment, 220-bp in length, and BARE-1, by two short fragments, each about $900 \mathrm{bp}$ in length (Additional file 1: Table S4).

A comparison of the DNA composition at the CENH3 locus in the progenitor species of each of the three genomes of cultivated wheat T. aestivum (ABD) and the corresponding genomes of hexaploid wheat itself yields a more complicated and perplexing result. Due to longer IS2, the CENH3 locus is twice as large in the A genome of T. aestivum as in the candidate progenitor species $T$. urartu. While the respective sets of retrotransposon copia families and LINE families in both species are absolutely identical (Additional file 1: Table S4), the ratio of gypsy: copia elements sharply increases in favor of gypsy, from 0.55 y Triticum urartu to 3.0 in the A genome of T. aestivum (Table 3), due to three Fatima elements, with one of them as a full-length entity. Here we note the presence of the copia family WIS, which is the commonest in various wheat species. A comparison of the CENH3 locus in the D genome of T. aestivum and in its candidate progenitor species, Aegilops tauschii, revealed an amazing similarity not only in the composition of retrotransposon families and LINE families, but also in the contribution each family makes and in the size of each intergenic spacer.

A comparison of the CENH3 locus in the B genome of T. aestivum and in its candidate progenitor species $A e$. speltoides yields quite a different result. The size of this locus in Ae. speltoides is $17.5 \mathrm{~kb}$, and its DNA composition is similar to that of the loci in Stipeae and Brachypodieae. IS2 contains mainly LINE, groups of simple repeats, and short fragments of DNA transposon families. The CENH3 locus in the B genome of T. aestivum is giant, 30 times as large as it is in the Ae. speltoides, which is because of an increase in the size of IS2 (Fig. 6b). IS2 is enriched for retrotransposon families, while the number of gypsy elements is only $54 \%$ higher than that of copia elements, which is in contrast to the IS2 situation in the A genome. IS2 of the B genome contains four fulllength copies of copia elements and five full-length copies of gypsy elements (Additional file 1: Table S4), along with numerous fragments of the same families, diverse in length and nested within one another. Additionally, there are numerous fragments of LINE and various DNA transposon families, diverse in length, too. A specific feature of the DNA composition in IS2 is the presence of 46 short fragments of the CACTA transposon family, totaling as few as $17,550 \mathrm{bp}$. CACTA is the commonest transposon family in wheat genomes; however, it is absent from the CENH3 locus in the $\mathrm{A}$ and $\mathrm{D}$ genomes. The size and DNA composition of the CENH3 locus in the B genome of T. aestivum are indicative of extensive recombination involving insertion of TEs during the evolutionary and breeding processes acting on this genome in $T$. aestivum. The insertion events had been taking place for a long period of time, as the presence of both full-length and truncated copies suggests.

\section{Discussion}

\section{Molecular mechanisms of CENH3 duplication}

Gene duplication is very common in plants, especially among angiosperms or flowering plants [49]. An important role has been attributed to gene duplications in generating evolutionary novelty and adaptation [50]. They come in either of two main ways: through polyploidization, when the whole genome is doubled (WGD), and due to local gene duplication or duplication of small sections of the genome, small-scale duplication (SSD). Several WGD events in the evolution of angiosperms have been described [51-53] and a nonrandom pattern of genome duplications has been shown to occur over time with many WGDs clustering around the Cretaceous-Paleogene extinction event about 66 million years ago [54]. WGDs are normally accompanied by subsequent loss of genes or more extended genome regions and genomic rearrangements [55]. Until now, it was known that the ancestors of the Triticeae species with two paralogous CENH3 genes (barley, rye, wheat) and the cereal species closest to them with one copy of CENH3 (rice, maize) split around $60 \mathrm{Mya}$ [32] and belong to different subfamilies. There is every reason to claim that the CENH3 locus, which consists of the syntenic genes CDPK2, $\alpha C E N H 3$ and $b Z I P$, is of ancient origin and emerged around 50 Mya (Fig. 1). In the maize genome, the CENH3 gene occurs as a single copy and has a different environment, which probably emerged due to deletions and rearrangements of a considerable part of the duplicated genome. In all Pooideae species that we have studied, $\beta C E N H 3$ resides within the locus, which excludes the WGD assumption.

Another hypothesis about the origin of several CENH3 paralogs stems from a vague understanding of how the CENH3 gene emerged in LECA (the last eukaryotic common ancestor). A study of the evolutionary history of 159 samples of histone $\mathrm{H} 3$ and $\mathrm{CENH} 3$ variants showed that 
the $\mathrm{H} 3$ variants evolved independently within the related species of almost all eukaryotic supergroups [1]. All core histone types are encoded in the genome of a basal dinoflagellate [1], although dinoflagellate chromatin is not organized into nucleosomes [56]. The analysis carried out by Postberg et al. [1] leaves open whether a protoCENH3 ancestral to all eukaryotic CENH3s had existed or whether extant CENH3s have multiple origins in eukaryotic evolution. The differences in the exon-intron structures of the genes encoding different variants make it even more difficult to trace the evolutionary origin of the histone $\mathrm{H} 3$ family. All variants of the canonical histone, H3.1, H3.2 and H3.3, share more than 95\% identity [4]; H3.3 genes revealing introns - three in P. virgatum [12], while canonical histone $\mathrm{H} 3$ (H3.1) has none. In most CENH3 gene duplications known in plant species, both paralogs have the same number of exons $[17,19,20]$, although their number varies across species. In these cases, duplication may have occurred due to homologous recombination involving unequal-crossing over or gene conversion, as has been shown for duplications of $\mathrm{Cid}$, a counterpart of CENH3 in Drosophila [57].

Our comparison of the structure of the CENH3 paralogs in cereals revealed differences in the exon-intron structure (Fig. 4), which resemble the differences in the structure of the variants of canonical histone $\mathrm{H} 3$ and obscure the mechanism of the duplications that have occurred in Pooideae evolution. Considering all the possibilities (WGDs, the independent origin of the paralogs from the corresponding variants of protoCENH3, SSD), we believe that the tandem duplication originating from unequal crossing-over is the most likely mechanism of duplication in the study species. This view is strongly supported by a small distance between the paralogs in Stipa and Brachypodium (Fig. 6). It is likely that duplications had more complex mechanisms in Stipa and Brachypodium species than in any other plant taxon $[17,19$, 20]. In cereals, the duplication was accompanied by splicing abnormalities, leading to changes in the exon-intron structure and long deletions in the $\mathrm{N}$-terminal domain of the protein $\beta C E N H 3$. The exon-intron structure of $\beta C E N H 3$ is preserved in all subsequent species (Fig. $4 \mathrm{~b}$ ). This is favored by negative (purifying) selection, which acts on both paralogs - to different extents, though. Although selective pressure exerted on $\beta C E N H 3$ is lower, it is still sufficient for the daughter copies and their exonintron structure to have been preserved for 35-40 Myr. Our argument is consistent with data on the structure of the centromere-specific histones in mosquito, mosqCid 1 and mosqCid2, which are subject to distinct selective pressures, have highly divergent $\mathrm{N}$-terminal tails and have been coretained for over $150 \mathrm{Myr}$ [58].
However, it is quite likely that DNA-mediated duplication is not the only mechanism for an increase in the number of $C E N H 3$ copies in cereal genomes. In $H$. vulgare, $\alpha C E N H 3$ and $\beta C E N H 3$ form part of the CENH3 locus and localize on chromosome $1 \mathrm{H}$. The emergence of yet another copy of $\beta C E N H 3$ on chromosome $6 \mathrm{H}$ due to an independent duplication is difficult to explain by the recombination mechanism. It is more logical to assume that this additional copy arouse as a result of an SSD event with the involvement of a messenger RNA intermediate, by analogy with the phenomenon observed and described in Arabidopsis thaliana embryogenesis [59]. Analysis of the functional divergence of a large number of duplicated genes in cereals [60] showed that neofunctionality often occurs in daughter copies and is associated with the mechanism driving RNA-mediated duplication in a way similar to observations in Drosophila [61]. The most likely explanation for this is that when this mechanism is a factor the daughter copy moves to other chromosomes and appears in a different genomic environment and, consequently, under a different regulatory system.

\section{Species-specific evolution of the CENH3 locus}

Our analysis of the genomic environment of the CENH3 genes in 23 Poaceae species suggests that the duplication that led to the new functional paralogs of the CENH3 gene first occurred in the tribes Stipeae and Brachypodieae. This is confirmed by the presence of only one copy of this gene in the preceding tribes in the phylogenetic trees, statistically significant evidence of diversifying selection of the $\beta C E N H 3$ genes on the branches of S. sibirica, S. breviflora and B. sylvaticum and the proximity of the paralogs at the CENH3 locus in these species. The subfamily Pooideae, which has species in the six tribes that we have used in our study, including Triticeae and Poeae, formed 40-45 Mya [62], while Brachypodieae split off 35 Mya [36, 62]. The exact age of the other three tribes, Nardeae, Meliceae and Stipeae, is unknown to us; what we know is that, according to Soreng et al. [22], they split off from their common Pooideae ancestor before Brachypodieae (Fig. 1). Because there is not a single Stipeae species with both a genomic and a transcriptome library developed for it, we have yet to be able to determine the exon-intron structure of the CENH3 paralogs. Thus, our assumption about an independent duplication of the CENH3 gene in Stipeae is based on the observation that its paralogs display much higher sequence homology in S. sibirica and S. breviflora than in any other Pooideae species (Table 2). In the ML tree, clusters with $\alpha$ CENH3 and $\beta C E N H 3$ in S. sibirica and S. breviflora lie adjacent to each other, indicating a high structural similarity between 
these CENH3 forms in these species (Fig. 3). The Stipeae species have specific amino acid motifs in $\beta C E N H 3$, which Brachypodieae, Triticeae and Poeae do not (Additional file 2: Fig. S4). The further evolution of the CENH3 locus from Brachypodium to cultivated species of barley and wheat displays a general tendency towards its expansion due to an increasing size of IS2 (Fig. 6b), which in the overwhelming majority of species correlates with increases in genome size. The main contributors to the increased size of IS2 and the CENH3 locus are TEs, primarily those in the main superfamilies of LTR-containing retrotransposons. This general tendency has specific features in Triticeae and Poeae and in each species within these tribes. These features can be more distinctly traced by considering Triticeae species, for their genomes are the best studied.

The $\beta C E N H 3$ inversion that we found in the CENH3 locus of the barley species is, in our experience, a Triticeae-only feature and occurred in their common ancestor approximately 15-16 Mya [63]. Invasion by mobile elements and concomitant rearrangements in the structure of the CENH3 locus took place in an independent way during the formation of each species' genome. As a result of these processes, we observe significant variations in the size of the locus, the set of TE families and the amount of changes in their structure. The handsdown champion in all parameters is the locus in the $B$ genome of T. aestivum, its size being almost $293 \mathrm{~kb}$, of which $285 \mathrm{~kb}$ is contributed by IS2 (Fig. 6b). Researchers consider five Aegilops L. species in the Sitopsis sectionAe. speltoides, Ae. longissima, Ae. sharonensis, Ae. bicornis and Ae. searsii-to be putative donors of the B genome in T. aestivum, and Ae. speltoides is often considered as one of the main donors [64]. However, as far as the CENH3 locus is concerned, with IS2 as short as $2.86 \mathrm{~kb}$, this species has contributed virtually nothing to IS2 in the B genome of T. aestivum. Our results tend to support the hypothesis of gene flow between all Aegilops L. species in the Sitopsis section and the B genome of wheat [65, 66]. It seems logical to assume that distant hybridization, which is considered as a stress factor that intensifies recombination, the multiplication and activity of TEs in the genomes, was the reason for such a rapid expansion of the CENH3 locus in the synthetic B genome and its enrichment of mobile elements. This assumption is also confirmed by the size of this locus in the cultivated barley $H$. vulgare and in the A genome of wheat $T$. aestivum, which is 1.75 and 1.83 times larger than that in their wild progenitors, $H$. spontaneum and $T$. urartu, respectively. However, a comparison of the structure of the CENH3 loci in the $\mathrm{D}$ genome of T. aestivum and in its putative progenitor Ae. tauschii shows a similarity in the size of the locus, intergenic spacers and the composition of TE families (Additional file 1: Table S4) and questions the universality of such a scenario. Additionally, the wheat $\mathrm{A}, \mathrm{B}$, and $\mathrm{D}$ genomes are estimated to have diverged at about the same time, between 2.5 and $4.5 \mathrm{Mya}$ [67], while allopolyploidization occurred 0.5 Mya $[67,68]$. While the size of IS2 is subject to substantial variation, it is really surprising to observe that the sizes of two other intergenic spacers of the CENH3 locus, IS1 and especially IS3, are relatively highly conserved. Only IS1 in the Poeae species D. glomerata and A. sativa, in which $\beta C E N H 3$ has undergone a duplication event, defies this tendency.

Analysis of the representation of TE families at the CENH3 locus in different species provides additional evidence in favor of the independent formation of this locus in different lineages. It has been shown many times that the gypsy-like retrotransposon families contribute about twice as much to the plant genomes as the copia-like families $[48,69,70]$. In the genomes we have looked into, only in the CENH3 locus of the A genome T. aestivum this ratio being equal to 3.0. In all the other genomes, the CENH3 locus is to varying degrees enriched for copialike families. This is best observed in the $H$. vulgare and $T$. urartu genomes, to which the copia families contribute, respectively, four times and twice as much as the gypsy families (Table 3). A large number of rearranged and truncated elements indicates that invasion of the CENH3 locus by TEs has occurred many times during the formation of each species' genome. For example, in IS2 of $H$. vulgare, fragments of the two most common copia-like elements, BARE1 and Maximus, make up about half of the locus, BARE1 being represented by two full-size copies. Maximus is not represented by full-size copies; however, six truncated fragments of this element in IS2 of $H$. vulgare (Additional file 1: Table S4) total nearly as much as two full-size copies of $B A R E 1$. It is most likely that Maximus invaded the CENH3 locus well before BARE1. The CENH3 locus in the wild progenitor of cultivated barley, $H$. spontaneum, whose genome is more ancient, is devoid of full-length copies of retrotransposons.

Analysis of the gradient of various classes of TEs in intergenic spacers revealed yet another interesting tendency. Intergenic spacers of the CENH3 locus in rice reveal simple repeats and short DNA fragments of nonautonomous transposons Harbinger and Mariner. The intergenic spacers in the loci in Stipeae and Brachypodieae species are composed of about the same classes of DNA sequences. Stipa sibirica has all these components and additionally non-LTR retrotransposon LINE. LTR retrotransposons appear in IS2 in Triticeae and Poeae, and their insertion sites begin and end several kilobases away from genes. This gradient of different classes of TEs 
in the vicinity of coding genes is-or at least appears to be-a common rule, because it is characteristic of the entire barley genome [48].

\section{Conclusion}

The following are the most pivotal stages in the emergence and formation of a two-component system of encoding the centromere-specific histone CENH3 in cereals. The syntenic group or the $C E N H 3$ locus with the $C E N H 3$ gene and the boundaries defined by CDPK2 and bZIP first appeared around 50 Mya in a common ancestor of the subfamilies Bambusoideae, Oryzoideae and Pooideae. This locus came to Pooideae, which split off ca. 40-45 Mya, with a single copy of CENH3 in the most ancient tribes Nardeae and Meliceae. The $\beta C E N H 3$ gene as a part of the locus appeared in the tribes Stipeae and Brachypodieae, which split off 35 Mya. The duplication was accompanied by splicing abnormalities, leading to changes in the exon-intron structure and long deletions in the $\mathrm{N}$-terminal domain of the protein $\beta$ CENH3. Purifying selection acts mostly on $\alpha \mathrm{CENH} 3 \mathrm{~s}$, while $\beta$ CENH3s and especially their NTT domains form more heterogeneous structures, in which clade-specific amino acid motifs are present. In barley species, the $\beta C E N H 3$ gene assumed an inverted orientation relative to $\alpha C E N H 3$ and the left-border gene, $C D P K 2$, was substituted with $L H C B-l$. A head-to-head orientation of the paralogs is a distinctive feature of all Triticeae species studied. As the evolutionary and domestication processes went on, the locus was growing in size due to an increasing distance between $\alpha C E N H 3$ and $\beta C E N H 3$. In turn, the distance increased due to a massive insertion of elements of the main LTR-containing retrotransposon superfamilies, gypsy and copia, without any evolutionary preference on either of them. A comparison of the molecular structure of this locus in the A, B and D subgenomes of the hexaploid wheat $T$. aestivum and in candidate donor species showed that invasion by transposable elements and concomitant rearrangements in the structure of the CENH3 locus took place independently even in evolutionarily closely related species.

\section{Materials and methods}

\section{Choosing cereal species for identification of CENH3} orthologs and paralogs in sequenced genomes

Cereal species were chosen for identification of orthologous and paralogous genes encoding centromeric histone CENH3 based on the positions these species take in the worldwide phylogenetic classification of the family Poaceae [22] and if their genome assemblies, whether full or partial, were annotated in GenBank (https://www. ncbi.nlm.nih.gov/nuccore/) and Phytozome (https:// phytozome-next.jgi.doe.gov/). A total of 23 species were included in the analysis (the list is given in Table 1); their phylogenetic relationships under the classification system of Soreng et al. [22] are presented in Fig. 1. If the databases had only partial genome assemblies (for Stipa sibirica and Lolium perenne), the available reads were downloaded from the SRA at NCBI (https://www.ncbi. nlm.nih.gov) and assembled into contigs (Additional files 3 and 4) using the assembly software Minia [26]. The search for $\mathrm{CENH} 3$ sequences in full-length genomic sequences or in the contigs assembled was done using TBLASTN or BLASTN from the AB-BLAST suite [71] (https://blast.advbiocomp.com). The sequence of the DNA encoding protein CENH3 or the sequence of this protein itself in a given species or in its nearest taxonomic neighbors was used as a query.

\section{Analysis of the CENH3 genes and transcripts in Brachypodium distachyon}

For the analysis of the genomic region containing the sequences of the CENH3 genes from the annotated genome of Brachypodium distachyon (GenBank NC_016132.3) and the structures of their potential transcripts, we used line Bd21 seeds (kindly provided by Dr. N. Collins, University of Adelaide, and Dr. Y. Shavrukov, Flinders University, Australia). Total RNA was extracted from young seedlings of Bd21 plants with TRI Reagent (MRC Inc., United States) and treated according to the manufacturers' recommendations with the DNA-free Kit (Thermo Fisher Scientific) containing DNase. RNA was reverse-transcribed to cDNA with the SuperScript IV Reverse Transcriptase (Thermo Fisher Scientific). Based on the annotated sequence of transcript LOC100830307, the following primers were designed for the putative paralogous genes: $5^{\prime}$-TGGCCCGCACGAAGCG $-3^{\prime}$ and $5^{\prime}$-CCTGTGCCCACTGATACGCC-3' ${ }^{\prime}$ (primers 1 and 2 for $\alpha C E N H 3)$; $5^{\prime}$-ATGGCTCGCACCAAGCA- $3^{\prime}$ and $5^{\prime}$-TGGATGGCCAAGAGATTCGC-3' (primers 3 and 4 for $\beta C E N H 3$ ). The following PCR conditions were used: $3 \mathrm{~min}$ at $95^{\circ} \mathrm{C}$ followed by 35 or 40 cycles at $94^{\circ} \mathrm{C}$ for $1 \mathrm{~min}, 59^{\circ} \mathrm{C}$ for $70 \mathrm{~s}$, and $72^{\circ} \mathrm{C}$ for $90 \mathrm{~s}$, with a final extension for $5 \mathrm{~min}$ at $72^{\circ} \mathrm{C}$. RT-PCR products were cloned using the InsTAclone PCR Cloning Kit (Thermo Fisher Scientific). Both strands of 15-20 clones of each CENH3 paralog were sequenced using the BigDye Terminator v3.1 Cycle Sequencing Kit and the ABI $3130 \times 1$ Genetic Analyzer (Applied Biosystems Inc., CA).

\section{Phylogenetic analysis}

The genomic sequences of $\alpha C E N H 3$ and $\beta C E N H 3$ obtained for 23 species and the deduced protein sequences were used for phylogenetic analysis. Multiple alignments were performed using Muscle [40] or Bali-Phy [41] to identify taxon- and species-specific features in the protein 
structures. Homology searches and pair-wise alignments were performed using FASTA [72]. The type of selective pressure acting on the CENH3 genes was determined by estimating the ratio of synonymous (Ks) to nonsynonymous (Ka) nucleotide substitution rates $(\omega)$. Estimation of $\omega$ from all-to-all pair-wise sequence comparisons was performed by KaKs_Calculator, Version 2.0 [46].

The evolutionary history was inferred by using the Maximum Likelihood method and JTT matrix-based model [42]. The tree with the highest log likelihood $(-4953.02)$ is shown. The percentage of the trees with the associated taxa clustered together is shown next to the branches. Initial tree(s) for the heuristic search was obtained automatically with Neighbor-Join and BioNJ algorithms applied to the matrix of pairwise distances estimated using a JTT model, and then by selecting the topology with the superior log likelihood value. Differences in evolutionary rates among sites were modeled using a discrete Gamma distribution (5 categories $(+G$, parameter $=1.0416)$ ). The tree was drawn to scale, with branch lengths measured in the number of substitutions per site. The analysis involved 45 amino acid sequences. The final dataset contained 200 positions. Evolutionary analyses were conducted in MEGA X [43]. Bootstrap values were calculated from at least 1000 replications. Amino acid sequences were aligned using MUSCLE with default settings.

Tests for selective pressure and positive selection on the individual branches of the phylogenetic trees were performed using aBSREL, which infers the optimized number of $\omega$ rate categories per branch [73]. Complementary transcripts of the $\alpha C E N H 3$ and $\beta C E N H 3$ sequences were codon-aligned using MUSCLE [40] and then uploaded to and analyzed on the DataMonkey server (http://datam onkey.org/).

\section{Determination of the exon-intron structure}

The sequences of the $\alpha C E N H 3$ and $\beta C E N H 3$ transcripts were determined using an approach similar to the one described above, which was used for the search for the sequences of these genes. In the absence of the wholegenome sequences of a given species, RNA-seq reads were downloaded from the SRA at NCBI (the accessions numbers are given in Additional file 1: Table S1) and assembled into transcript contigs using Minia [26] and/or Trinity [74]. In the assemblies, CENH3 transcripts were found and analyzed using TBLASTN. The lists of the assembled mRNA $\alpha C E N H 3$ and mRNA $\beta C E N H 3$ sequences are presented in Additional files 5 and 6 . The lists of the deduced amino acid sequences of the $\alpha \mathrm{CENH} 3$ and $\beta \mathrm{CENH} 3$ proteins are presented in Additional files 7 and 8.
To determine the exon-intron structure of the $\alpha C E N H 3$ and $\beta C E N H 3$ genes, RNA-seq reads from the SRA at NCBI were mapped onto the corresponding reference genome sequences or the assembled contigs of genome sequences (if the whole-genome reference sequences were not available) using HISAT2 [75] and STAR [76]. The output files in SAM format were visualized and subjected to a primary analysis using Geneious 11.0.2 (http:// www.geneious.com) [77]. Analyses of the exon-intron structure, splice sites and read coverage were performed using StringTie [78] and Trinity [74].

\section{Identification of the CENH3 locus in cereal genomes}

The primary search for and localization of the genome copies of $\alpha C E N H 3$ and $\beta C E N H 3$ were carried out on the best-studied and annotated genomes, T. urartu and $O$. sativa. We downloaded these genomes from NCBI and found homologous sequences in T. urartu spaced $20 \mathrm{~kb}$ apart, using BLASTN with the sequences of $\alpha C E N H 3$ and $\beta C E N H 3$ genes in rye as queries [70]. Rice was found to have homology only with $\alpha C E N H 3$. We examined the genomic environment of the $C E N H 3$ genes and found that upstream of $\beta C E N H 3$ are the $L H C B-l$ gene, which encodes chlorophyll a-b binding protein 3C (EMS53958) in T. urartu, and the CDPK2 gene, which encodes calcium-dependent protein kinase 2 (XP_015638740.1) in $O$. sativa. Downstream of $\alpha C E N H 3$ both species have a $b Z I P$ transcription factor-encoding gene (XP_015638736.1). We used the genome sequences of $\alpha C E N H 3, \beta C E N H 3$ and the neighboring genes as markers of the CENH3 locus and identified these syntenic genes in the genome sequences of the other cereal species. The composition of repeated DNA sequences in the CENH3 locus in different species was determined using the Viridiplantae RepeatMasker program and database [73]. The genes neighboring to CENH3 were identified by homology with the sequences of $T$. urartu and $O$. sativa using TBLASTX at NCBI or, locally, BLASTN.

\section{Abbreviations}

LTR: Long terminal repeat; Mya:: Million years ago; SSD: Small-scale duplication.; WGD: Whole-genome duplication.

\section{Supplementary Information}

The online version contains supplementary material available at https://doi. org/10.1186/s12870-021-03264-3.

Additional file 1 Table $\mathbf{S 1}$. Sources of the genome sequences and transcripts of the CENH3 locus genes used for analysis and contig assembly. Table S2. Estimates of $\omega$ values from all-to-all pair-wise comparison of aCENH3 coding sequences. Values of Ka and Ks are shown above the diagonal. The $\omega$ values are shown below the diagonal followed by $P$-values in brackets. Table S3. Values of Ka and Ks are shown above the diagonal. The $\omega$ values are shown below the diagonal followed by P-values in brackets. 
Values of Ka and Ks are shown above the diagonal. The $\omega$ values are show below the diagonal followed by P-values in brackets. Values $\omega>1$ are highlighted in bold. Table S4. The presence of the most well-represented transposable element families in ISs in Triticeae species. The total number of base pairs for given superfamily of transposable elements is shown in bold. The values after the family names are (1) the percentage abundance of the family fragments in the superfamily and (2) the numbers of the family fragments. The number of asterisks ${ }^{*}$ ) represents the number of full-length copies of the element.

Additional file 2 Fig. S1. CENH3 nucleotide sequences from barley species virtually translated into proteins and aligned. Accession numbers are given in Table S1. The sequence of canonical histone H3.3 in H. vulgare was retrieved from GenBank (accession number 769350). Fig. S2. An amino acid alignment inferred using the genomic sequences of three Brachypodium species (accession numbers are given in Table S1). Asterisks stand for stop codons in the sequences of sample 2 and sample 4. The aberrant transcript of B. stacei_1 was predicted by mapping the transcriptome of B. stacei (SRR4094449, SRR4094447, DRR090154) onto its genome using HISAT2 [75] followed by an analysis of the transcripts with StringTie [78]. Fig. S3. Experimental confirmation of the presence of a stop codon in exon 2 of BCENH3 in B. distachyon. 1. A schematic of locus LOC100830307 retrieved from GenBank. 2. A schematic of a 1776-bp transcript from the locus. Exons appear as white finger-post arrows. 3. A schematic of sequences in locus LOC100830307 homologous to BCENH3 and aCENH3 in the rye genome [25]. 4. Products of RT-PCR with primers of 1 and 2 for aCENH3 and primers 3 and 4 for $\beta C E N H 3$. Arrows point to the primer positions, stop codons are shown by cross. The upper scale shows the dimensions in bp. Fig. S4. A multiple alignment of the amino acid sequences of the CENH3 proteins using the Bali-Phy program [41]. BAli-Phy is software that estimates multiple sequence alignments and evolutionary trees from DNA, amino acid, or codon sequences. It uses likelihood-based evolutionary models of substitutions and insertions and deletions to place gaps. Redelings showed that BAli-Phy had 3.5 times fewer alignment errors than MUSCLE and MAFFT on simulated data [41]. For convenience, the alpha and beta forms are grouped into two separate blocks: 1-26 (aCENH3) and 27-45 (BCENH3). Amino acid residues identical in all species are shown as white letters on the black background. Amino acid motifs specific for certain tribes and genera are contained within rectangular boxes with different border colors. Fig. S5. Branch-site (BSREL) analysis of positive selection for the aCENH3 (a) and $\beta C E N H 3$ (b) [47]. The upper scale indicates the length of branches. Branch lengths are scaled to the expected number of substitutions per amino acid and branch colors indicate the strength of selection ( $\mathrm{dN} / \mathrm{dS}$ or $\omega$ ). Green, positive selection $(\omega>5)$; black, purifying selection $(\omega=0)$; gray, neutral evolution $(\omega=1)$. The proportion of each color represents the fraction of the sequence undergoing the corresponding class of selection. Branch thickness reflects the amount of statistical support for evolution under episodic diversifying positive selection as determined by BSREL. The tables show the branches and nodes in which BSREL found evidence of episodic diversifying selection at $P<0.05$.

Additional file $\mathbf{3}$ The DNA sequence of the CENH3 locus of Stipa sibirica assembled in this study.

Additional file 4 The DNA sequence of the CENH3 locus of Lolium perenne assembled in this study.

Additional file $\mathbf{5}$ The sequences of mRNA aCENH3 assembled for this study.

Additional file 6 The sequences of mRNA $\beta C E N H 3$ assembled for this study.

Additional file 7. The amino acid sequences of aCENH3 assembled for this study.

Additional file 8. The amino acid sequences of $\beta$ CENH3 assembled for this study.

\section{Acknowledgments}

The authors thank Dr. N. Collins, University of Adelaide, and Dr. Y. Shavrukov, Flinders University, Australia for providing us by the line Bd21 seeds of B. distachyon.

\section{Authors' contributions}

AVV conceived the project; EAE and AVV designed the study; EAE and EVE contributed to the investigation; EAE, EVE and AVV analyzed the data; AVV wrote the manuscript. All authors read and approved the final manuscript.

\section{Funding}

This work was supported by the Russian fundamental scientific research program (project № 0246-2021-0014); molecular analysis was supported by grant of Russian Foundation for Basic Research (project № 20-04-00699), phylogenetic and evolutionary analysis were funded by the Russian Science Foundation (project № 19-14-00051).

\section{Availability of data and materials}

The datasets underlying this article are available as supplementary information in the article and in its online supplementary material. The data will be shared on request to the corresponding author.

\section{Declarations}

Ethics approval and consent to participate

Not applicable.

\section{Consent for publication}

Not applicable.

\section{Competing interests}

The authors declare that they have no conflict of interest.

\section{Author details}

${ }^{1}$ Institute of Cytology and Genetics, SB RAS, Novosibirsk 630090, Russia. ${ }^{2}$ Institute of Molecular and Cellular Biology, SB RAS, Novosibirsk 630090, Russia.

${ }^{3}$ Novosibirsk State University, Novosibirsk 630090, Russia.

Received: 21 March 2021 Accepted: 12 October 2021

Published online: 18 November 2021

\section{References}

1. Postberg J, Forcob S, Chang W-J, Lipps HJ. The evolutionary history of histone $\mathrm{H} 3$ suggests a deep eukaryotic root of chromatin modifying mechanisms. BMC Evol Biol. 2010;10:259.

2. Iwasaki W, Miya Y, Horikoshi N, Osakabe A, Taguchi H, Tachiwana H, et al. Contribution of histone $\mathrm{N}$-terminal tails to the structure and stability of nucleosomes. FEBS Open Bio J. 2013;3:363-9.

3. Earnshaw WC, Rothfield N. Identification of a family of human centromere proteins using autoimmune sera from patients with scleroderma. Chromosoma. 1985;91:313-21.

4. Allshire RC, Karpen $\mathrm{GH}$. Epigenetic regulation of centromeric chromatin: old dogs, new tricks? Nat Rev Genet. 2008;9:923-37.

5. Black BE, Jansen LET, Maddox PS, Foltz DR, Desai AB, Shah JV, et al. Centromere identity maintained by nucleosomes assembled with histone $\mathrm{H} 3$ containing the CENP-A targeting domein. Mol Cell. 2007;25:309-22.

6. Tachiwana H, Kagawa W, Shiga T, Osakabe A, Miya Y, Saito K, et al. Crystal structure of the human centromeric nucleosome containing CENP-A. Nature. 2011:476:232-5.

7. Hasson D, Panchenko T, Salimian KJ, Salman MU, Sekulic N, Alonso A, et al. The octamer is the major form of CENP-A nucleosomes at human centromeres. Nat Struct Mol Biol. 2013;20:687-95.

8. Fukagawa T, Earnshaw WC. The centromere: chromatin foundation for the kinetochore machinery. Dev Cell. 2014;30:496-508.

9. Malik HS, Henikoff S. Phylogenomics of the nucleosome. Nat Struct Mol Biol. 2003;10:882-91.

10. Ingouff M, Berger F. Histone $\mathrm{H} 3$ variants in plants. Chromosoma. 2010;119:27-33.

11. Okada T, Endo M, Singh MB, Bhalla PL. Analysis of the histone H3 gene family in Arabidopsis and identification of the male-gamete-specific variant AtMGH3. Plant J. 2005;44:557-68. 
12. Miao J, Frazier T, Huang $L$, Zhang $X$, Zhao B. Identification and characterization of switchgrass histone $\mathrm{H} 3$ and $\mathrm{CENH} 3$ genes. Front Plant Sci. 2016;7:979

13. Wells D, Hoffman D, Kedes L. Unusual structure, evolutionary conservation of non-coding sequences and numerous pseudogenes characterize the human $\mathrm{H} 3.3$ histone multigene family. Nucl Acids Res. 1987; 15:2871-89.

14. Talbert PB, Masuelli R, Tyagi AP, Comai L, Henikoff S. Centromeric localization and adaptive evolution of an Arabidopsis histone $\mathrm{H} 3$ variant. Plant Cell. 2002;14:1053-66.

15. Zhong CX, Marshall JB, Topp C, Mroczek R, Kato A, Nagaki K, et al. Centromeric retroelements and satellites interact with maize kinetochore protein CENH3. Plant Cell. 2002;14:2825-36.

16. Nagaki K, Cheng ZK, Yang SO, Talbert PB, Kim M, Jones KM, et al. Sequencing of a rice centromere uncovers active genes. Nat Genet. 2004;36:138-45.

17. Kawabe A, Nasuda S, Charlesworth D. Duplication of centromeric histone H3 (HTR12) gene in Arabidopsis halleri and A. lyrata, plant species with multiple centromeric satellite sequences. Genetics. 2006;174:2021-32.

18. Wang G, He Q, Liu F, Cheng Z, Talbert PB, Jin W. Characterization of CENH3 proteins and centromere-associated DNA sequences in diploid and allotetraploid Brassica species. Chromosoma. 2011;120:353-65.

19. Neumann P, Pavlıkova Z, Koblızkova A, Fukova I, Jedlickova V, NovakP, et al. Centromeres off the hook: massive changes in centromere size and structure following duplication of $\mathrm{CenH} 3$ gene in Fabeae species. Mol Biol Evol. 2015;32:1862-79.

20. Finseth FR, Dong Y, Saunders A, Fishman L. Duplication and adaptive evolution of a key centromeric protein in Mimulus, a genus with female meiotic drive. Mol Biol Evol. 2015;32:2694-706.

21. Chalupska D, Lee HY, Faris JD, Evrard A, Chalhoub B, Haselkorn R, et al. Acc homoeoloci and the evolution of wheat genomes. Proc Natl Acad Sci U S A. 2008;105:9691-6

22. Ishii T, Juranic M, Maheshwari S, Bustamante F, Vogt M, Salinas-Gamboa R, et al. Unequal contribution of two paralogous CENH3 variants in cowpea centromere function. Commun Biol. 2020;3:775.

23. Soreng RJ, Peterson PM, Romaschenko K, Davidse G, Zuloaga FO, Judziewicz EJ, et al. A worldwide phylogenetic classification of the Poaceae (Gramineae). J Syst Evol. 2015;53:117-37.

24. Sanei M, Pickering R, Kumke K, Nasuda S, Houben A. Loss of centromeric histone $\mathrm{H} 3$ (CENH3) from centromeres precedes uniparental chromosome elimination in interspecific barley hybrids. Proc Natl Acad Sci U S A. 2011;108:498-505.

25. Yuan J, Guo X, Hu J, Lv Z, Han F. Characterization of two CENH3 genes and their roles in wheat evolution. New Phytol. 2015;206:839-51.

26. Evtushenko EV, Elisafenko EA, Gatzkaya SS, Lipikhina YA, Houben A, Vershinin AV. Conserved molecular structure of the centromeric histone CENH3 in Secale and its phylogenetic relationships. Sci Rep. 2017;7:17628.

27. Leitch IJ, Johnston E, Pellicer J, Hidalgo O, Bennett MD. Plant DNA C-values Database (release 7.1). 2019. https://cvalues.science.kew.org/

28. Dolezel J, Bartoš J, Voglmayr H, Greilhuber J. Nuclear DNA content and genome size of trout and human. Cytom Part A. 2003;51:127-8.

29. Li W, Shi C, Li K, Zhang Q-J, Tong Y, Zhang Y, et al. The draft genome sequence of herbaceous diploid bamboo Raddia distichophylla. bioRxiv. The preprint server for biology. Genomics. 2020. doi: https://doi. org/https://doi.org/10.1101/2020.04.27.064089.

30. Pohl RW, Davidse G. Chromosome numbers of Costa Rican grasses. Brittonia. 1971;23:293-324.

31. Chikhi R, Rizk G. Space-efficient and exact de Bruijn graph representation based on a bloom filter. Algorithms Mol Biol. 2013;8:22.

32. Hunziker JH, Wulff AF, Soderstrom TR. Chromosome studies on Anomochloa and other Bambusoideaea (Gramineae). Darwiniana. 1989;29:41-5.

33. Brassac J, Blatner FR. Species-level phylogeny and polyploid relationships in Hordeum (Poaceae) inferred by next-generation sequencing and in silico cloning of multiple nuclear loci. Syst Biol. 2015;64:792-808.

34. Feng C, Yuan J, Bai H, Liu Y, Su H, Liu Y, et al. The deposition of CENH3 in maize is stringently regulated. Plant J. 2020;102:6-17.

35. Ishii T, Karimi-Ashtiyani R, Banaei-Moghaddam AM, Schubert V, Fuchs J, Houben A. The differential loading of two barley CENH3 variants into distinct centromeric substructures is cell type- and development-specific. Chromosom Res. 2015;23:277-84.
36. International Brachypodium Initiative. Genome sequencing and analysis of the model grass Brachypodium distachyon. Nature. 2010;463:763-8.

37. Scholthof K-B, Irigoyen S, Catalan P, Mandadi KK. Brachypodium: a monocot grass model genus for plant biology. Plant Cell. 2018;30:1673-94.

38. Maheshwari S, Tan EH, West A, Franklin FC, Comai L, Chan SW. Naturally occurring differences in CENH3 affect chromosome segregation in zygotic mitosis of hybrids. PLoS Genet. 2015;11:e1004970.

39. Li Y, Zuo S, Zhang Z, Li Z, Han J, Chu Z, et al. Centromeric DNA characterization in the model grass Brachypodium distachyon provides insights on the evolution of the genus. Plant J. 2018;93:1088-101.

40. Edgar RC. MUSCLE: multiple sequence alignment with high accuracy and high throughput. Nucl Acids Res. 2004;32:1792-7.

41. Redelings BD. BAli-Phy version 3: Model-based co-estimation of alignment and phylogeny. bioRxiv. 2020. https://doi.org/10.1101/2020.10.10 334003.

42. Jones DT, Taylor WR, Thornton JM. The rapid generation of mutation data matrices from protein sequences. Comput Appl Biosci. 1992;8:275-82.

43. Kumar S, Stecher G, Li M, Knyaz C, Tamura K. MEGA X: Molecular evolutionary genetics analysis across computing platforms. Mol Biol Evol 2018;35:1547-1549.

44. Davis Jl, Soreng RJ. Phylogenetic structure in the grass family (Poaceae) as inferred from chloroplast DNA restriction site variation. Am J Bot. 1993;80:1444-54

45. Karimi-Ashtiyani R, Ishii T, Niessen M, Stein N, Heckmann S, Gurushidze $\mathrm{M}$, et al. Point mutation impairs centromeric $\mathrm{CENH} 3$ loading and induces haploid plants. Proc Natl Acad Sci U S A. 2015;112:11211-6.

46. Zhang Z, Li J, Zhao XQ, Wang J, Wong GK, Yu J. KaKs_Calculator: calculating $\mathrm{Ka}$ and $\mathrm{Ks}$ through model selection and model averaging. Genomics Proteomics Bioinformatics. 2006;4:259-63.

47. Smith MD, Wertheim JO, Weaver S, Murrell B, Scheffler K, Kosakovsky Pond SL. Less is more: an adaptive branch-site random effects model for efficient detection of episodic diversifying selection. Mol Biol Evol. 2015;32:1342-53.

48. Wicker $\mathrm{T}$, Gundlach $\mathrm{H}$, Schulman $\mathrm{AH}$. The repetitive landscape of the barley genome. In: Stein N, Muehlbauer JG, editors. The barley genome. Cham: Springer; 2018. p. 123-38.

49. Flagel LE, Wendel JF. Gene duplication and evolutionary novelty in plants. New Phytol. 2009;183:557-64.

50. Van de Peer Y, Mizrachi E, Marchal K. The evolutionary significance of polyploidy. Nat Rev Genet. 2017;18:411-24.

51. Paterson A, Bowers J, Chapman B. Ancient polyploidization predating divergence of the cereals, and its consequences for comparative genomics. Proc Natl Acad Sci U S A. 2004;101:9903-8.

52. Cui L, Wall PK, Leebens-Mack JH, Lindsay BG, Soltis DE, Doyle JJ, et al. Widespread genome duplications throughout the history of flowering plants. Genome Res. 2006;16:738-49.

53. Jiao Y, Wickett NJ, Ayyampalayam S, Chanderbali AS, Landherr L, Ralph PE, et al. Ancestral polyploidy in seed plants and angiosperms. Nature. 2011:473:97-100

54. Vanneste K, Baele G, Maere S, Van de Peer Y. Analysis of 41 plant genomes supports a wave of successful genome duplications in association with the cretaceous-Paleogene boundary. Genome Res. 2014;24:1334-47.

55. Vanneste $K$, Van de Peer $Y$, Maere S. Inference of genome duplications from age distributions revisited. Mol Biol Evol. 2013;30:177-90.

56. Saldarriaga JF, McEwan ML, Fast NM, Taylor FJ, Keeling PJ. Multiple protein phylogenies show that Oxyrrhis marina and Perkinsus marinus are early branches of the dinoflagellate lineage. Int J Syst Evol Microbiol. 2003:53:355-65.

57. Kursel LE, Malik HS. Recurrent gene duplication leads to diverse repertoires of centromeric histones in Drosophila species. Mol Biol Evol. 2017;34:1445-62.

58. Kursel LE, Welsh FC, Malik HS. Ancient coretention of paralogs of Cid centromeric histones and Cal1 chaperons in mosquito species. Mol Biol Evol. 2020;37:1949-63.

59. Tadini L, Ferrari R, Lehniger M-K, Mizzotti C, Moratti F, Resentini F, et al. Trans-splicing of plastid rps 12 transcripts, mediated by AtPPR4, is essential for embryo patterning in Arabidopsis thaliana. Planta. 2018;248:257-65.

60. Jiang $X$, Assis R. Rapid functional divergence after small-scale duplication in grasses. BMC Evol Biol. 2019;19:97.

61. Assis R, Bachtrog D. Neofunctionalization of young duplicate genes in Drosophila. Proc Natl Acad Sci U S A. 2013;110:17409-14. 
62. Bossolini E, Wicker T, Knobel PA, Keller B. Comparison of orthologous loc from small grass genome Brachipodium and rice: implications for wheat genomics and grass genome annotation. Plant J. 2007;49:704-17.

63. Bernhardt N, Brassac J, Kilian B, Blattner FR. Dated tribe-wide whole chloroplast genome phylogeny indicates recurrent hybridizations within Triticeae. BMC Evol Biol. 2017;17:141

64. Kilian B, Ozkan H, Deusch O, Effgen S, Brandolini A, Kohl J, et al. Independent wheat $B$ and $G$ genome origins in outcrossing Aegilops progenitor haplotypes. Mol Biol Evol. 2007:24:217-27.

65. El Baidouri M, Murat F, Veyssiere M, Molinier M, Flores R, Burlot L, et al. Reconciling the evolutionary origin of bread wheat (Triticum aestivum). New Phytol. 2017;213:1477-86.

66. Bernhardt N, Brassac J, Dong X, Willing E-V, Poskar CH, Kilian B. Blattner FR genome-wide sequence information reveals recurrent hybridization among diploid wheat wild relatives. Plant J. 2020;102:493-506.

67. Huang S, Sirikhachornkit A, Su X, Faris J, Gill B, Haselkorn R, et al. Genes encoding plastid acetyl-CjA carboxylase and 3-phosphoglycerate kinase of the Triticum/Aegilops complex and the evolutionary history of polyploid wheat. Proc Natl Acad Sci U S A. 2002;99:8133-8.

68. Dvorak J, Akhunov ED, Akhunova AR, Deal KR, Luo MC. Molecular characterization of a diagnostic DNA marker for domesticated tetraploid wheat provides evidence for gene flow from wild tetraploid wheat to hexaploid wheat. Mol Biol Evol. 2006;23:1386-96.

69. Wicker T, Sabot F, Hua-Van A, Bennetzen JL, Capy P, Chalhoub B, et al. A unified classification system for eukaryotic transposable elements. Nature Rev Genet. 2007;8:973-82

70. Evtushenko EV, Levitsky VG, Elisafenko EA, Gunbin KV, Belousov Al, Šafář $J$, et al. The expansion of heterochromatin blocks in rye reflects the co-amplification of tandem repeats and adjacent transposable elements. BMC Genomics. 2016;17:337.

71. Gish W. 2019. https://blast.advbiocomp.com

72. Pearson WR, Lipman DJ. Improved tools for biological sequence comparison. Proc Natl Acad Sci U S A. 1988;85:2444-8.

73. Smit AFA, Hubley R, Green P. RepeatMasker Open-4.0. 2013-2015, http:// www.repeatmasker.org

74. Grabherr MG, Haas BJ, Yassour M, Levin JZ, Thompson DA, Amit I, et al. Full-length transcriptome assembly from RNA-seq data without a reference genome. Nature Biotechnol. 2011;29:644-52.

75. Kim D, Langmead B, Salzberg SL. HISAT: a fast spliced aligner with low memory requirements. Nat Methods. 2015;12:357-60.

76. Dobin A, Davis CA, Schlesinger F, Drenkow J, Zaleski C, Jha S, et al. STAR: ultrafast universal RNA-seq aligner. Bioinformatics. 2013;29:15-21.

77. Kearse M, Moir R, Wilson A, Stones-Havas S, Cheung M, Sturrock S, et al. Geneious basic: an integrated and extendable desktop software platform for the organization and analysis of sequence data. Bioinformatics. 2012;28:1647-9.

78. Pertea M, Pertea GM, Antonescu CM, Chang T-C, Mendell JT, Salzberg SL. StringTie enables improved reconstruction of a transcriptome from RNAseq reads. Nature Biotechnol. 2015;33:290-5.

\section{Publisher's Note}

Springer Nature remains neutral with regard to jurisdictional claims in published maps and institutional affiliations.
Ready to submit your research? Choose BMC and benefit from:

- fast, convenient online submission

- thorough peer review by experienced researchers in your field

- rapid publication on acceptance

- support for research data, including large and complex data types

- gold Open Access which fosters wider collaboration and increased citations

- maximum visibility for your research: over 100M website views per year

At BMC, research is always in progress.

Learn more biomedcentral.com/submissions 